\title{
Shear response of 3D non-woven carbon fibre reinforced composites
}

\author{
Lucas R. Meza ${ }^{1}$, Jim M.J. Schormans ${ }^{2}$, Joris J.C. Remmers² ${ }^{2}$ Vikram S. Deshpande ${ }^{1^{*}}$ \\ ${ }^{I}$ Department of Engineering, University of Cambridge, \\ Trumpington Street, Cambridge CB2 1PZ, UK. \\ ${ }^{2}$ Department of Mechanical Engineering, Eindhoven University of Technology, \\ P.O. Box 513, 5600 MB Eindhoven, Netherlands.
}

\begin{abstract}
We experimentally and numerically investigated the shear response of a three-dimensional (3D) non-woven carbon fibre reinforced epoxy composite with three sets of orthogonal tows and approximately equal fibre volume fractions in the orthogonal directions. Shear tests on two orientations of dogbone specimens showed significant strain hardening and an increasing unloading stiffnesses with increasing applied strain. Unloading was also accompanied by considerable strain recovery, with X-ray tomographic scans revealing minimal damage accumulation in specimens until near final failure at shear strains in excess of $50 \%$. To understand the origins of this unusual mechanical response of the 3D carbon fibre composites, we developed a micro-mechanical model wherein all tows and matrix pockets in the composite are explicitly considered. The tows were modelled using a pressure-dependent crystal plasticity approach to capture texture evolution under large deformations and the model replicated many of the experimental observations with a high degree of fidelity. Importantly, the model illustrated the role of the $3 \mathrm{D}$ architecture in not only suppressing delamination but also enhancing the strain hardening response due to a 3D confinement effect of the tow architecture On the other hand, a model wherein the tows were modelled using an anisotropic Hill plasticity framework (absent plastic spin) failed to replicate the observed strain hardening response or capture the associated strain recovery upon unloading. This highlights the importance of accounting for the evolution of the material substructure within the tows of these high ductility 3D composites. The results of this work illustrate the unique mechanical behaviour of 3D nonwoven fibre composites and provide insight into how 3D fibre architecture can be used to enhance the mechanical performance of fibre composites.
\end{abstract}

Keywords: 3D CFRP composites, crystal plasticity, shear hardening, texture evolution, plastic incompatibility

*Corresponding author. E-mail address: vsd@eng.cam.ac.uk. 


\section{Introduction}

The remarkable strength-to-weight performance of fibre composites, in particular carbon fibre reinforced polymer composites (CFRPs), has led to their extensive application in the fields of aerospace, automotive, and maritime engineering (Jacob et al., 2002; Mouritz et al., 2001; Poe et al., 1999). A majority of these fibre composites have a two-dimensional (2D) architecture, and are fabricated as unidirectional or woven laminated sheets. Such laminated composites have many mechanical drawbacks, including poor interlaminar strength and a tendency for catastrophic failure under many loading states (Carolan et al., 2017; Daniel et al., 2009; Farley and Jones, 1992; Jelf and Fleck, 1992; Vogler and Kyriakides, 1999).

Fibre composites can be designed with a 3D architecture in an attempt to overcome some of these limitations (Huang et al., 2017; Mouritz et al., 1999). Some of these designs include braided tubes and nozzles, multilayer woven sheets, stitched composites and beams with complex cross sections (Kamiya et al., 2000; Kazemahvazi et al., 2016; Schmidt et al., 2004; Umer et al., 2017; Yudhanto et al., 2015). These developments have successfully enabled 3D composites not only to overcome many of the vulnerabilities of laminated composites but also to exhibit many novel mechanical properties including enhanced damage tolerance, resistance to compressive microbuckling failure, and a capacity for large ductility and energy absorption (Cox et al., 1996, 1994, 1992; Das et al., 2018; Evans and Adler, 1978). The properties of 3D composites make them attractive for mechanical design purposes, but understanding the micromechanical origins of their behaviour and in particular developing numerical models that capture these responses has proved difficult.

Numerical models for the plasticity and failure behaviour of 3D composites generally incorporate either a maximum stress criterion or a criterion based on the second invariant of the stress tensor (Ansar et al., 2011). These are often used in conjunction with continuum damage models that capture the degradation of the mechanical properties. For example, Tan et al. (2000) employed a maximum stress criterion to determine the tensile failure limit of a microlevel 3D unit cell and then incorporated these results into a macro-level model that accurately reproduced the longitudinal failure strengths of a 3D composite. However, the model gave poor predictions of the transverse strengths. The "binary model" of McGlockton et al. (2003) illustrated the role of inter-fibre friction and interlocking in enhancing the toughness of 3D composites. The micro-buckling of $2 \mathrm{D}$ and $3 \mathrm{D}$ woven composites has also received considerable attention. Song et al. (2007) and Huang and Waas (2009) developed a transversely isotropic plasticity model to capture the buckling response of a braided and z-pinned composite respectively, while Das et al. (2017) modelled the progressive microbuckling in a 3D nonwoven composite using a transversely isotropic Hill plasticity criterion for the tows. A key feature of all these models is that while they typically are able to capture responses in specific directions, they lose accuracy under multi-axial loading (Ansar et al., 2011).

Techniques for modelling of unidirectional (UD) and laminated composites are relatively well established. They typically involve three interconnected features: (i) a constitutive model for predicting the deformation and associated stress-states, (ii) a damage initiation criterion and (iii) a damage evolution law that degrades the constitutive properties and sets the final material failure. In their most common form, the constitutive models are either anisotropic elastic or elasto-plastic and employ an anisotropic quadratic yield criteria like the Hill model (Hill, 1948); see for example Beissel (2014), Choi et al. (2018) and Hasanyan and Waas (2018). The stress predictions from these constitutive models are then used to estimate the onset of damage. The most commonly used failure criterion was pioneered by Hashin (1980), and since then there have been numerous modifications and extensions. For example, Puck and Schürmann 
(1998) developed micro-mechanically motivated failure envelopes for UD composites that capture the tension-compression asymmetry and shear-dominated failure of fibre composites. The accuracy and applicability of their method has been validated through a detailed series of micromechanical fibre-level simulations (Naya et al., 2017). The LaRC04 criterion proposed by Pinho et al. (2005) extends such models by accounting for non-linear kinematics within failure zones such as kink bands and been validated for both laminate failure and microbuckling (Llorca et al., 2011; Naya et al., 2017). In the simplest models, the initiation of damage is used to identify failure, although the more advanced implementations use damage evolution models such as the combined plastic and smeared crack model (Camanho et al., 2013; Vogler et al., 2013) or the continuum shear damage model by Tan and Falzon (2016). An additional complication in composite modelling is the pressure dependency of the plastic deformation of the tows and matrix. This can have a pronounced effect on the properties of a composite (Wisnom, 1995; Shin and Pae, 1992), and generally results in an approximately linear increase in shear strength with hydrostatic pressure (Hine et al., 2005; Vyas et al., 2011). While many advanced plasticity and failure models incorporate this effect (Pinho et al., 2005; Puck and Schürmann, 1998; Vogler et al., 2013), simpler models often ignore this dependency.

One of the most commonly neglected phenomena in the fibre composite constitutive models is the spin of the material substructure (fibres) with respect to the overall material rotation. Most traditional laminated and UD composites fail at relatively low strains (on the order of a few percent). Consequently, the constitutive models discussed above typically ignore the evolution of the internal substructure within tows and use plasticity models that do not accounting for plastic spin (Hasanyan and Waas, 2018). Some attempts to include fibre rotation within tow constitutive models have been reported (Sun and Zhu, 2000; Mandel et al., 2015), but it is generally considered acceptable to ignore these effects in traditional 2D laminated composites especially when predicting the overall stress-states. However, recent work by Das et al. (2018) has shown that 3D composites display a large ductility, sometimes on the order of $20 \%$, bringing into question the validity of ignoring such effects for $3 \mathrm{D}$ composites.

In this work, we investigate the shear response of a non-woven, orthogonally oriented (noobed) carbon fibre reinforced polymer (CFRP) composite (Khokar, 2002, 2001). We show that this composite has a large ductility and an associated strain hardening response that is unlike traditional UD and 2D laminated composites. The origins of the unusual behaviour are experimentally probed via strain mapping using digital image correlation (DIC) and X-ray computed tomography (XCT). Numerical models are presented to help elucidate the underlying deformation and failure mechanisms that govern the behaviour of the noobed composite. Through these calculations, we demonstrate that accurately modelling the evolution of the material substructure within the tows (i.e. the texture of the tows) as well as accounting for the pressure dependent yield of the matrix and tows are critical to capturing the large strain behaviour of these 3D composites. 

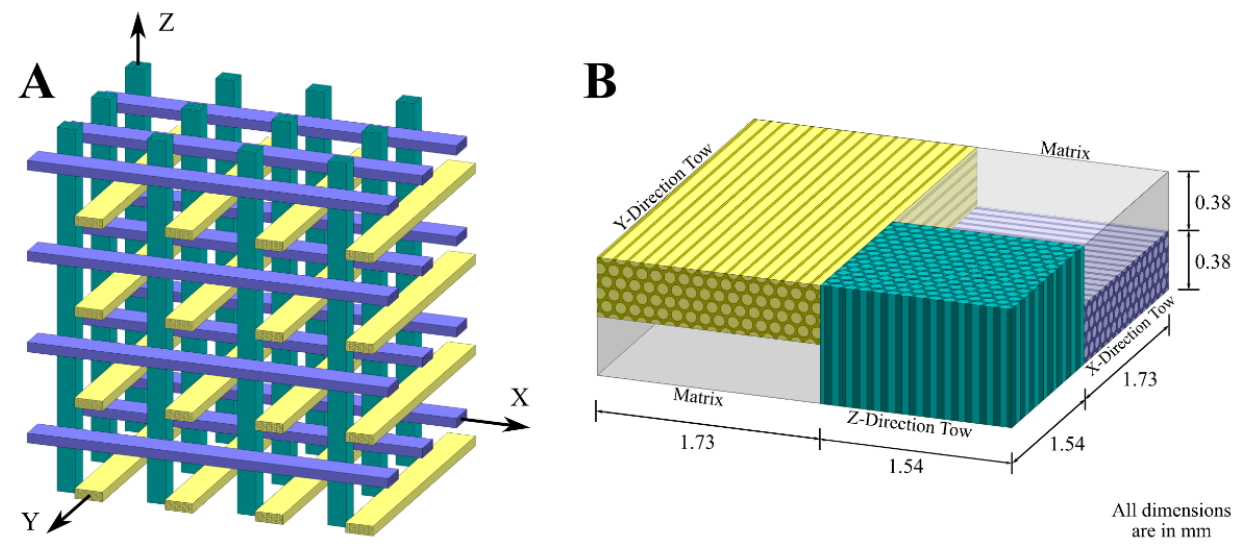

Figure 1: (A) Sketch of the orthogonal non-woven yarns in the 3D noobed fabric. This fabric is infused with epoxy to create the $3 \mathrm{D}$ noobed composite, wherein the yarns are then referred to as tows. (B) Sketch of the unit cell of the composite employed in this study with dimensions indicated in $\mathrm{mm}$. The co-ordinate system $(X, Y, Z)$ is indicated and the colour scheme used for the $X, Y, Z$-tows and matrix is replicated throughout the manuscript.

\section{Experimental protocol}

The aim of the experimental study is to measure the shear response of the $3 \mathrm{D}$ noobed composites and investigate the dominant deformation and failure mechanisms. We first briefly describe the manufacture of these composites, followed by the measurement protocols and then proceed to discuss observations of the shear behaviour.
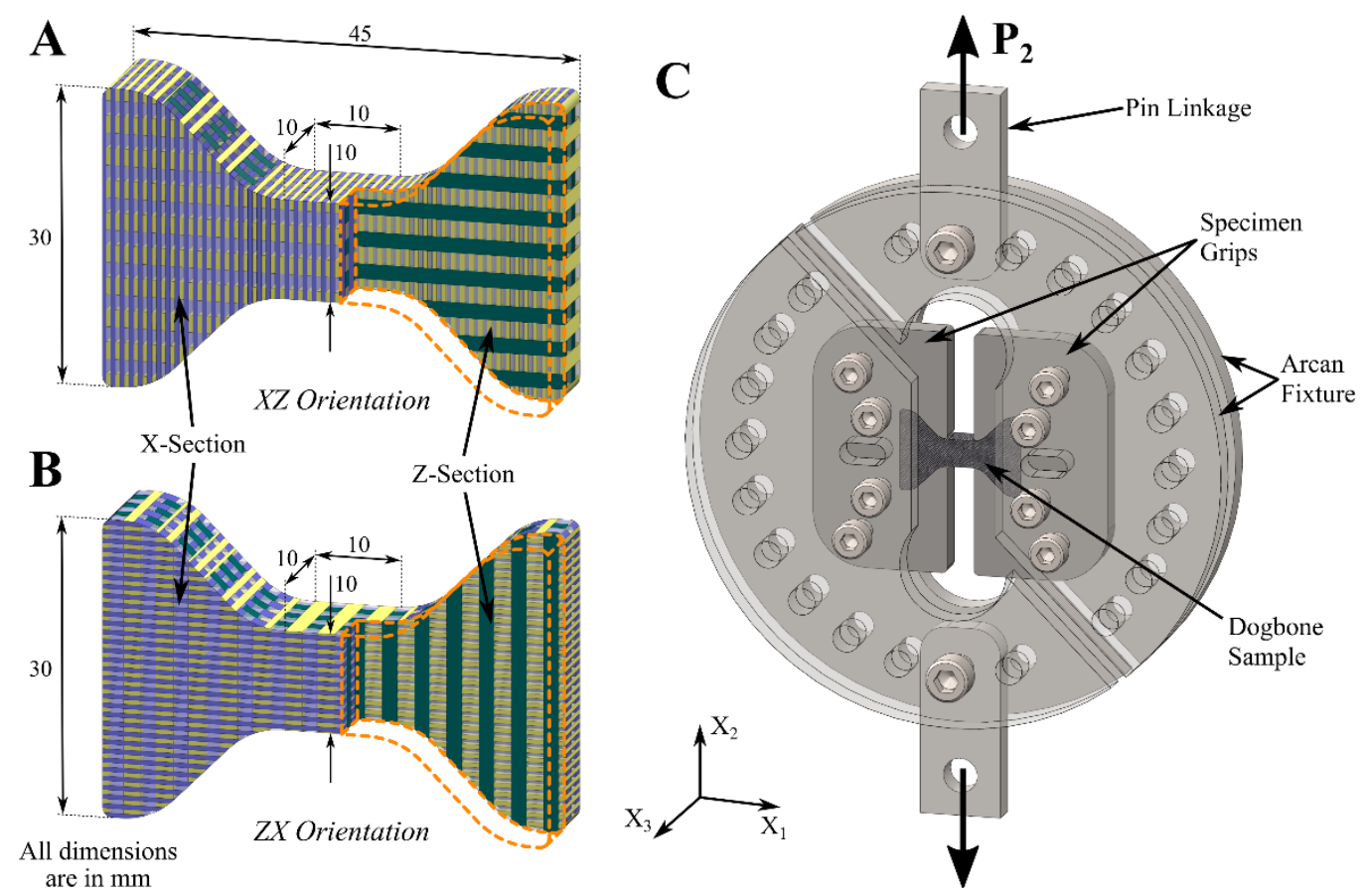

Figure 2: Sketch of the dogbone specimens in the (A) $X Z$ and (B) $Z X$ orientation with leading dimensions marked in $\mathrm{mm}$. The global co-ordinate system $X_{i}$ used to define the loading is also indicated. (C) Sketch of the Arcan setup used for imposing shear loading.

\subsection{Materials and manufacture}

In the noobing process, which is fundamentally different from traditional weaving, knitting or braiding, linear sets of yarns in three orthogonal directions are bound together to produce a 3D layerless and crimpless fabric with pure matrix filling the interstitial gaps between tows 
(Fig. 1). Since the yarns do not interlace, interloop or intertwine, the fabrics are referred to as noobed (the acronym NOOB standing for Non-interlacing, Orientating Orthogonally and Binding) (Khokar, 1997). There are a variety of noobing processes, and readers are referred to (Khokar, 2002) for a detailed discussion.

The composites used in this study were manufactured ${ }^{1}$ employing the method developed by (Khokar, 2012) using T700S carbon fibre tow bundles (Toray composite Materials America Inc., USA) and infused with NM FW 3070 epoxy (Nils Malmgren $\mathrm{AB}$, Sweden). With $(X, Y, Z)$ forming a Cartesian co-ordinate system, the composite comprises an array of $Z$-tows bound together by $X$ and $Y$-tows. The $Z$-tows contain $\sim 24 \mathrm{k}$ fibres while the $X$ - and $Y$-tows have $\sim 12 \mathrm{k}$ fibres. The overall volume fraction of fibres in the infused 3D composite was $V_{f} \approx 42 \%$, with $V_{f}^{Z}=40 \%$ by volume fibres present in the $Z$-tows and $V_{f}^{X}=V_{f}^{Y}=62 \%$ by volume fibres in the $X$ - and $Y$-tows. The composites were manufactured in blocks of size $\sim 200 \mathrm{~mm} \times$ $125 \mathrm{~mm} \times 50 \mathrm{~mm}$ and test specimens were cut from these blocks.

Shear tests were conducted using the dogbone specimen as sketched in Fig. 2. Slices of thickness $10 \mathrm{~mm}$ were first cut using a diamond saw from the as-manufactured noobed blocks, and then dogbone specimens of dimension labelled in Fig. 2 were waterjet cut from these slices. Some post-cut polishing was performed using 120-grit SiC sandpaper to ensure high quality finished edges. The gauge section of the dogbone specimens was approximately $10 \times 10 \times 10 \mathrm{~mm}$. A global co-ordinate system $X_{i}$ is defined as shown in Fig. 2 with the $X_{1}$ direction along the dogbone length and $X_{3}$ in the through-thickness direction of the specimen. Specimens were cut in two orientations from the noobed blocks and subjected to a shear strain $\Gamma_{21}$. In both specimen orientations, the $Y$-tows are along $X_{3}$-direction with the two orientations defined as:

(i) $\quad X Z$ Orientation: Shear displacement is applied parallel to the $X$-tows with the $Z$ tows aligned in $X_{1}$-direction in the undeformed configuration (Fig. 2A).

(ii) $\quad Z X$ Orientation: Shear displacement is applied parallel to the $Z$-tows with the $X$ tows aligned in the $X_{1}$-direction in the undeformed configuration (Fig 2B).

\footnotetext{
${ }^{1}$ The noobed fabrics were supplied by Biteam AB, Danderydsgatan 23, SE-114 26 Stockholm, Sweden but are now available from Fureho AB, Segloravägen 6, SE-504 64 Borås, Sweden.
} 


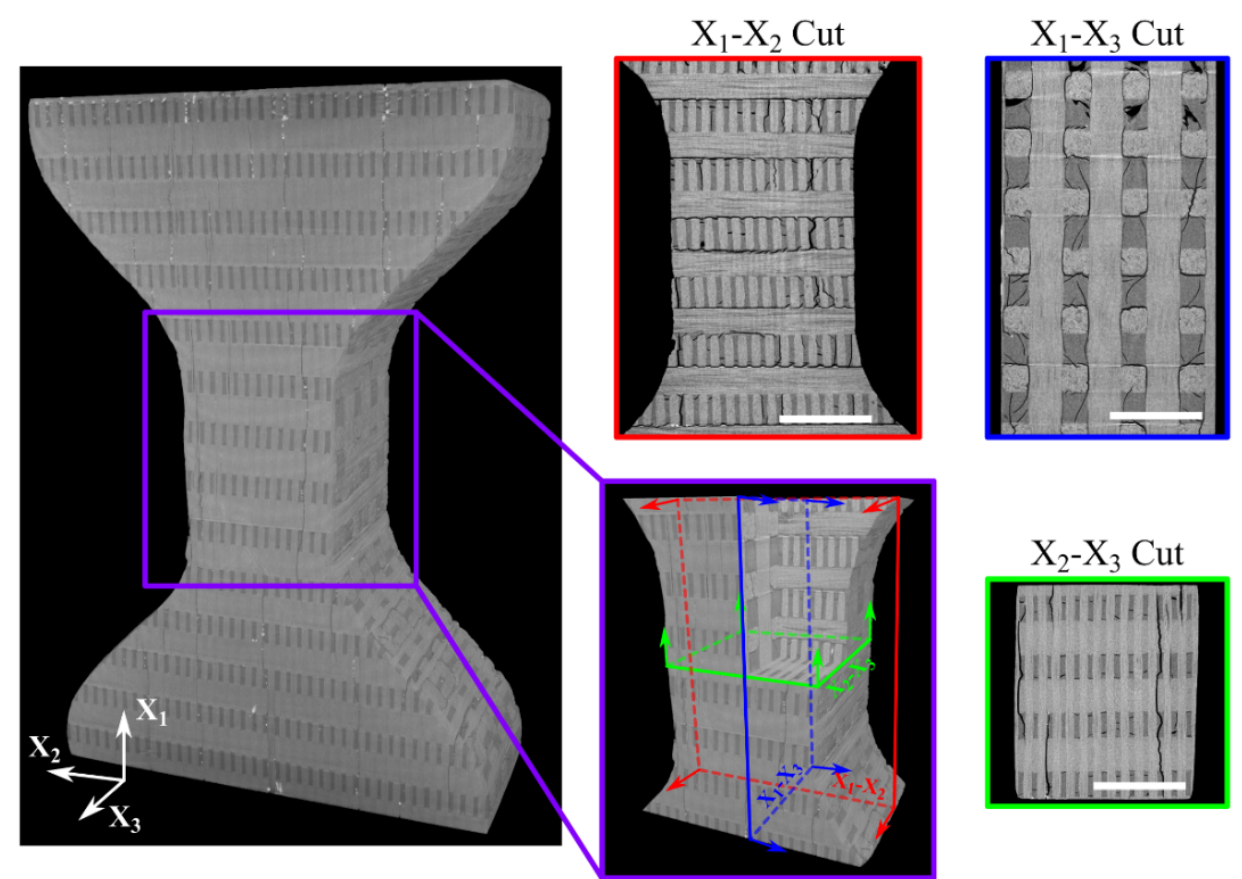

Figure 3: X-ray tomographic scans of a specimen in the $Z X$ orientation. The insets show the gauge section along with various cross-sections. All scale bars $=5 \mathrm{~mm}$.

\subsection{Microstructure of noobed composite}

A series of XCT images illustrating the microstructure of the as-infused noobed composite specimen are shown in Fig. 3. The XCT images were taken using a X-Tek XTH 225ST system (Nikon Metrology UK Ltd.). Based on images taken at different locations within specimens, we inferred that the $X$ and $Y$-tows have rectangular cross-sections of dimension $0.38 \times 1.73 \mathrm{~mm}$. By contrast, the $Z$-tows have approximately a square cross-section of size $1.54 \mathrm{~mm}$. A unit cell (absent defects) based on these measurements is sketched in Fig. 1B. From these dimensions, it is apparent that the specimens used in this study comprised at-least three unit cells in every direction. More detailed micrographs and characterisation of the microstructural defects are given in Appendix A. In brief, there are two main types of defects:

(a) Tow and fibre waviness: The $X$ - and $Y$-tows had a global tow-level waviness with amplitudes $\sim 0.11 \mathrm{~mm}$ and $\sim 0.06 \mathrm{~mm}$ in the two orthogonal directions perpendicular and parallel to the length, respectively of the rectangular tow cross-section. This waviness resulted in the fibre volume fraction in the $X$ and $Y$-tows varying between $58 \%$ and $68 \%$ along the tow length, with an average $V_{f}^{X}=V_{f}^{Y}=62 \%$. The $Z$-tows had a negligible tow-level waviness but had significant fibre-level waviness within the tows (see Appendix A).

(b) Matrix cracks: Cracks in the form of debonded regions between tows can be observed in the images of the as-infused but untested composites (Fig. 3). They appear to initiate between tows and extend into the matrix pockets. We hypothesize that these cracks arise during cooling after matrix infusion due to the mismatch in thermal expansion coefficients between tows in the different directions and between the tows and the matrix.

\subsection{Test protocol}

Experiments were conducted in screw-driven mechanical test machine with an Arcan type fixture (Arcan et al., 1978) used to apply shear loading (Fig. 2C). This type of a setup is commonly used to measure the shear response of composites (Cognard et al., 2011). The setup applied primarily a simple shear loading by imposing a displacement $\delta$ in the $X_{2}$-direction. However, unlike typical single or double-lap shear tests, the Arcan setup also permitted axial 
displacement of the specimen in the $X_{1}$-direction such that the axial load $P_{1} \approx 0$ throughout the loading. In the Arcan setup the load $P_{2}$, conjugated to $\delta$, was measured directly via the load cell of the test machine and used to define the applied shear stress. At-least 8 tests were conducted in each case to confirm the repeatability of the measurements. In all cases, differences of less than 5\% were observed between repeated measurements. This high reproducibly, unlike that in traditional 2D composites, is associated with the strongly strain hardening response of these 3D composites as we shall discuss in detail subsequently.

Strain distributions over the specimen surface were inferred via Digital Image Correlation (DIC) using an ARAMIS 12M 3D-DIC setup (GOM GmbH). In addition to providing distributions of the strains within the specimen, the DIC data was used to determine the imposed shear displacement $\delta$ by measuring the average relative displacements of five material points located at the outer edge of the gauge section of the specimens. This method of measuring $\delta$ eliminated the influence of compliance within the Arcan fixture. The specimens were loaded by imposing a displacement rate $|\dot{\delta}|=0.3 \mathrm{~mm} \mathrm{~min}^{-1}$, which corresponds to an applied shear strain rate $\dot{\Gamma}_{21}=|\dot{\delta}| / \ell=5 \times 10^{-4} \mathrm{~s}^{-1}$ based on a specimen gauge length of $\ell=$ $10 \mathrm{~mm}$. The corresponding nominal shear stress $\mathcal{T}_{21}$ was then defined as $\mathcal{T}_{21} \equiv P_{2} / A_{c}$ where $A_{c}=100 \mathrm{~mm}^{2}$ is the cross-sectional area of the gauge section of the specimen. Three types of tests were performed:

(i) Specimens were taken to moderate $(30-45 \%)$ shear strains and then fully unloaded to enable inspection of deformation and damage evolution via X-ray tomography. The loading stiffness was estimated from the slope of the stress versus strain curve and the shear strength $\mathcal{T}^{Y}$ was defined as the shear stress at a $0.2 \%$ offset shear strain.

(ii) Specimens were loaded monotonically until the onset of complete failure. These tests were used to measure the entire shear stress versus strain curves up to the point of specimen failure.

(iii) Specimens were cyclically loaded and unloaded to investigate the evolution of damage via changes in the specimen stiffness. In each cycle, a strain increment of $\Delta \Gamma_{21} \approx 3.5 \%$ was imposed and the specimen was then unloaded to a shear stress $\mathcal{T}_{21}=1 \mathrm{MPa}$. These cycles were repeated until the total accumulated shear strain reached $\Gamma_{21} \approx 35 \%$. The unloading stiffness during each cycle was estimated by fitting a $5^{\text {th }}$ order polynomial to the unloading stress versus strain data and then calculating the slope of the curve at the initiation of unloading.

\section{Summary of experimental measurements}

We now summarize the key measurements and observations of the shear response of the noobed composites with an emphasis on their hardening response and ability to retain structural integrity up to large applied shear strains. The measurements will be discussed in the context of the evolution of damage within the composites as observed via X-ray tomography (videos of selected DIC measurements and X-ray tomographs are in the online supplementary). 

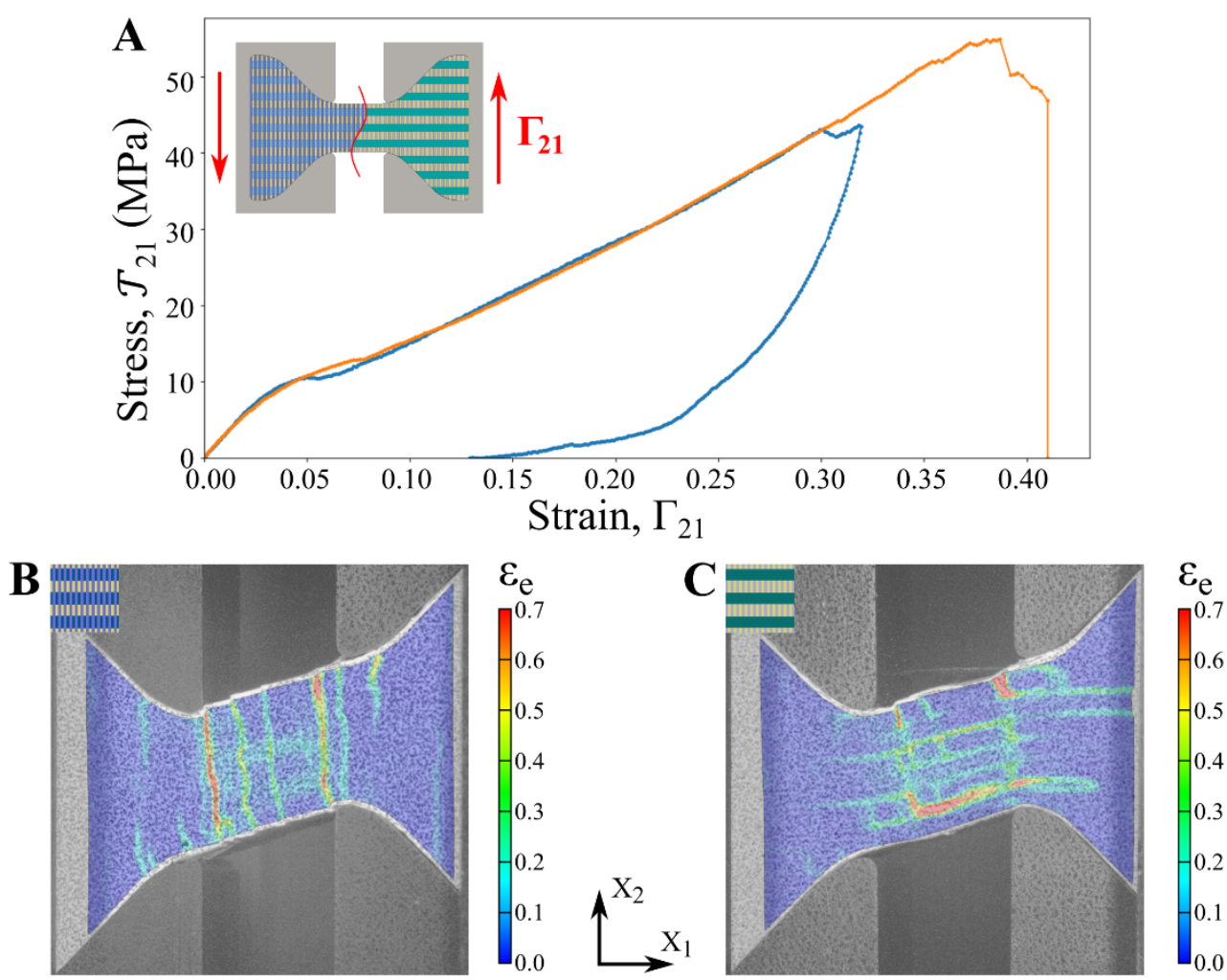

Figure 4: The shear response in the $X Z$ orientation. (A) The measured $\mathcal{T}_{21}$ versus $\Gamma_{21}$ responses from two separate measurements including one for a specimen taken to complete fracture. Measurements of the distribution of the effective strain $\varepsilon_{e}$ on (B) the $X$-section and (C) the $Z$-section of the specimens loaded to $\Gamma_{21} \approx 30 \%$. The specimen sketches in the inset use the colour scheme from Fig. 1.

\subsection{Shear response in the $X Z$ orientation}

The measured $\mathcal{T}_{21}$ versus $\Gamma_{21}$ responses from two representative $X Z$ orientation experiments are plotted in Fig. 4A, showing (i) a specimen loaded to $\Gamma_{21} \approx 32 \%$ then unloaded and (ii) a specimen loaded to complete failure. After an initial elastic phase with a shear modulus $G_{21} \approx$ $265 \mathrm{MPa}$, the specimens yielded at $\mathcal{T}_{21}^{Y} \approx 8 \mathrm{MPa}$ and subsequently displayed a linear hardening response with a hardening modulus $h_{21} \approx 130 \mathrm{MPa}$. This hardening continued until the onset of catastrophic failure due to the tensile fracture of the $Z$-tows at $\Gamma_{21}^{\text {ult }} \approx 42 \%$ and $\mathcal{T}_{21}^{\text {ult }} \approx$ $55 \mathrm{MPa}$, i.e. the ultimate shear strength is approximately 7 times higher than the initial yield strength. By contrast, unloading from $\Gamma_{21} \approx 32 \%$ resulted in significant recovery, with the shear stress reducing to zero at $\Gamma_{21} \approx 15 \%$, constituting an approximate $50 \%$ strain recovery.

We shall discuss the deformation and recovery mechanisms in the context of both the X-ray observations and the numerical models in Section 5. Here it suffices to say that large shear deformations in the $X Z$ specimen orientation lead to: (i) plastic straining of the tows and matrix pockets, and (ii) elastic straining of the tows primarily in the fibre direction. This elastic straining is one of the central causes of both the observed hardening and the recovery upon unloading. Continued elastic tensile straining of the tows results in catastrophic tensile rupture of the $Z$-tows and consequently the specimens as well.

\subsubsection{Deformation modes and X-ray tomography observations}

To visualize the deformation and failure modes, we performed both X-ray CT scans and DIC measurements. X-ray CT sections of the specimen in the $X Z$ orientation are included in Fig. 5 both prior to mechanical testing and after unloading from $\Gamma_{21} \approx 32 \%$. Two sections are shown 
in the $X_{1}-X_{2}$ plane: (i) the $X$-section, which exposes the $X$-and $Y$-tows, and (ii) the $Z$-section, which exposes the $Z$ - and $Y$-tows. In both views, cracks are clearly seen prior to testing, with cracks along the $X_{2}$-direction running between the $X$ - and $Y$-tows (seen in the $X$-section) and cracks along the $X_{1}$-direction running along the $Z$-tows (seen in the $Z$-section). Despite the direction of the applied shear loading, most of the $X_{2}$-direction cracks remained dormant during loading. Cracks along the $X_{1}$-direction tended to coalesce leading to greater debonding along the Z-tows after testing. Intriguingly, there was little to no visible accumulation of damage in areas of the specimen that were not pre-cracked.

To further quantify these observations, we conducted DIC measurements on specimens cut such that the $X_{1}-X_{2}$ surface of the specimen had either an $X$ - or $Z$-section exposed. To parameterise the surface deformations, we define a scalar effective strain measure $\varepsilon_{e} \equiv$ $\sqrt{(2 / 3) \sum_{i=1}^{2} \varphi_{i}^{2}}$ where $\varphi_{i}$ is given in terms of the principal stretches $\Lambda_{i}$ as $\varphi_{i} \equiv \ln \left(\Lambda_{i}\right)$. Spatial distributions of $\varepsilon_{e}$ are shown for the $X$ - and $Z$-sections in Figs. 4B and 4C, respectively at an applied specimen strain $\Gamma_{21} \approx 30 \%$. In these DIC snapshots, large localised vertical and horizontal bands of deformation with $\varepsilon_{e}>60 \%$ are seen on the $X$ and $Z$-sections respectively, mirroring the pre-existing crack patterns in the XCT sections (Fig. 5). Moderate strains of $\varepsilon_{e} \approx$ $20 \%$ are also observed at the tow-tow interfaces. Strain away from the pre-cracked regions and the tow interfaces remained small, suggesting that that majority of the deformation occurs at the interfaces between the tows and between the tows and the matrix pockets. We note here that DIC is a surface measurement technique that is heavily influenced by the numerical interpolation schemes employed: these strain measurements should therefore be used to estimate the locations of concentrated deformations rather than to provide measures of the true strains within each constituent phase of the composite.

$\mathrm{X}$-Ray $\mathrm{CT}$ images of the $X$ - and $Z$-sections of failed $X Z$ orientation specimens are included in Figs. $6 \mathrm{~A}$ and $6 \mathrm{~B}$, respectively. Failure is clearly seen to occur due to the tensile rupture of the $Z$-tows, accompanied with a debonding of the $X$ and $Y$-tows along the $X_{2}-X_{3}$ plane. Tow level pull-out accompanies the fracture of $Z$-tows, resulting in a zig-zag failure surface reminiscent of fracture surfaces that arise during fibre pull-out in unidirectional fibre composites (Hull and Clyne, 1996). Away from the fracture surfaces, there is no clear evidence of additional damage except additional bending of the $Z$-tows due to the large imposed shear strains. 


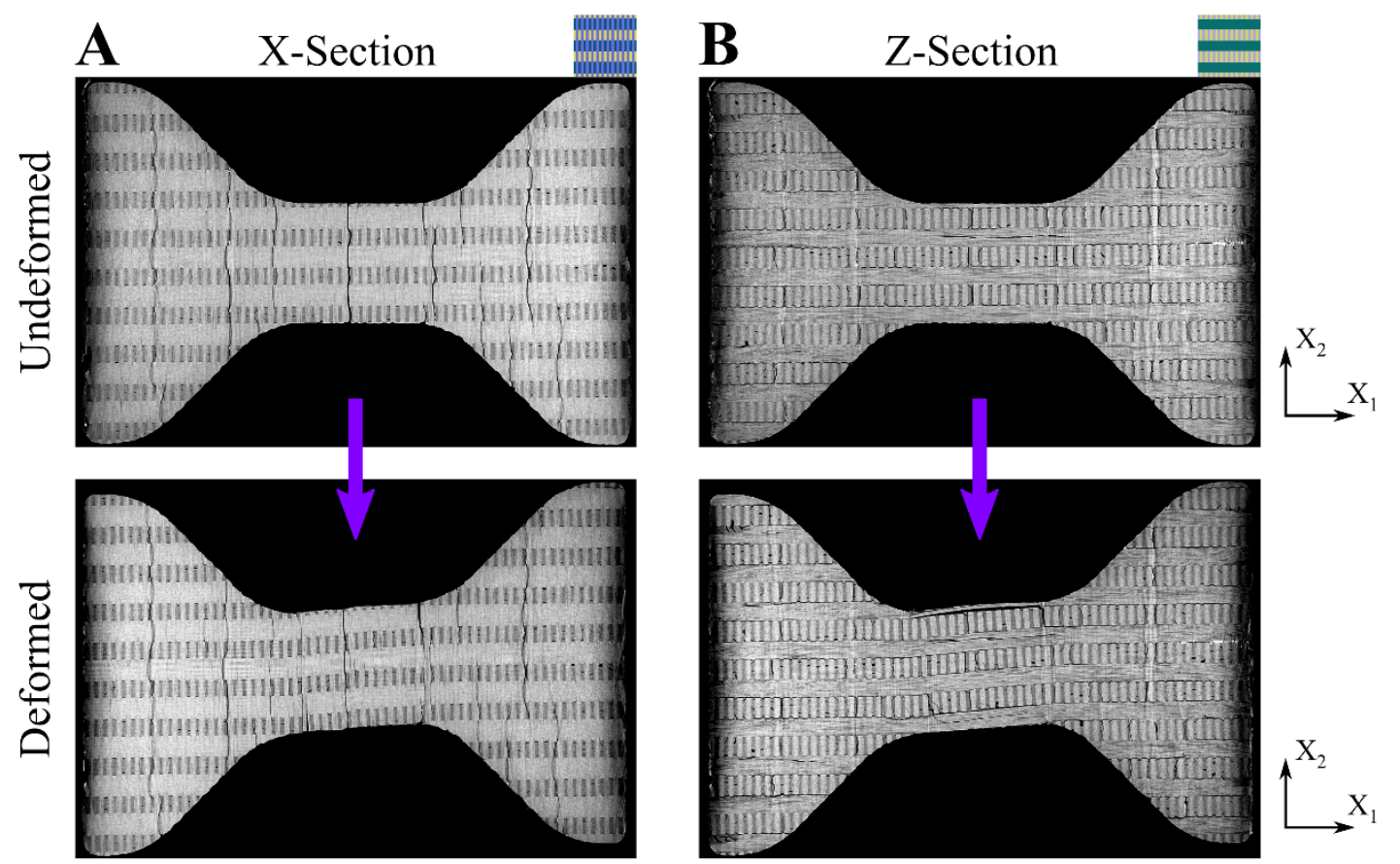

Figure 5: X-ray tomographic images of the (A) $X$-sections and (B) $Z$-sections of the specimen in the $X Z$ orientation. Images are shown of both the undeformed specimens and the specimen unloaded after application of a shear strain $\Gamma_{21} \approx 30 \%$. The sketches indicate the section of the specimen using the colour scheme from Fig. 1.
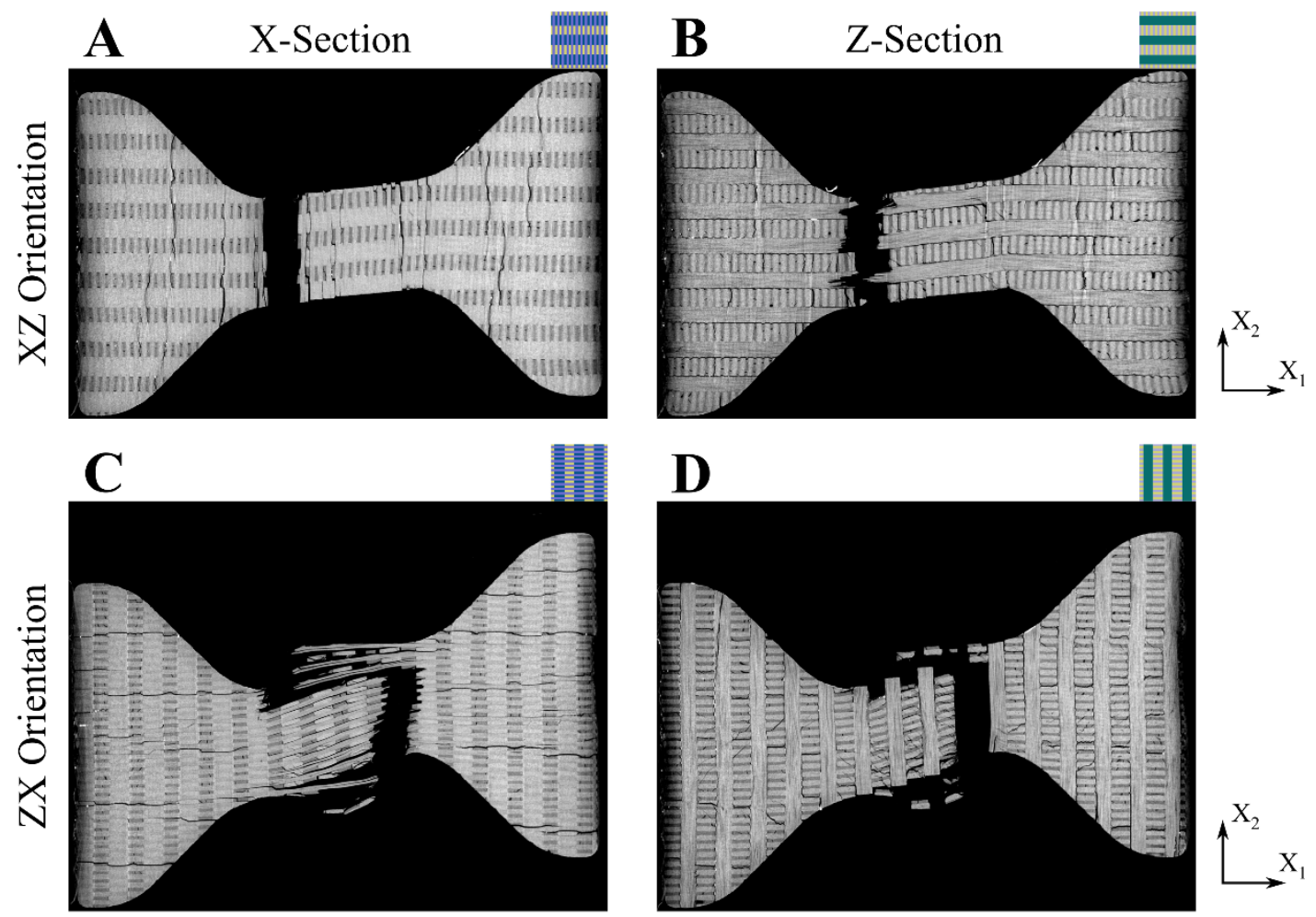

Figure 6: $X$-ray tomographic images of the failed sections of the specimens. (A) $X$-section, (B) $Z$ section of the specimen in the $X Z$ orientation, and (C) $X$-section, (D) $Z$-section of the specimen in the $Z X$ orientation. The sketches indicate the section of the specimen using the colour scheme from Fig. 1. 


\subsection{Shear response in the $Z X$ orientation}

The measured $\mathcal{T}_{21}$ versus $\Gamma_{21}$ responses from two representative $Z X$ orientation experiments are plotted in Fig. 7A, showing (i) a specimen loaded to $\Gamma_{21} \approx 32 \%$ then unloaded and (ii) a specimen loaded to complete failure. The responses are qualitatively similar to those of the $X Z$ orientation and comprise an initial elastic regime with a shear modulus $G_{21} \approx 380 \mathrm{MPa}$ followed by yielding with a strength $\mathcal{T}_{21}^{Y} \approx 12 \mathrm{MPa}$ and subsequent hardening. The hardening response is not strictly linear but rather there is a gradual increase in the hardening modulus up to $\Gamma_{21} \approx 48 \%$. Straining beyond this point results in a reduced hardening rate presumably due to development of damage within the specimens. Ultimate failure occurs at a strain of $\Gamma_{12}^{\text {ult } \approx}$ $70 \%$ and an associated strength of $\mathcal{T}_{21}^{\text {ult }} \cong 125 \mathrm{MPa}$, which is more than 10 times than the initial yield strength. Thus, specimens in the $Z X$ orientation have a higher ultimate failure strain and strength compared to the $X Z$ orientation.
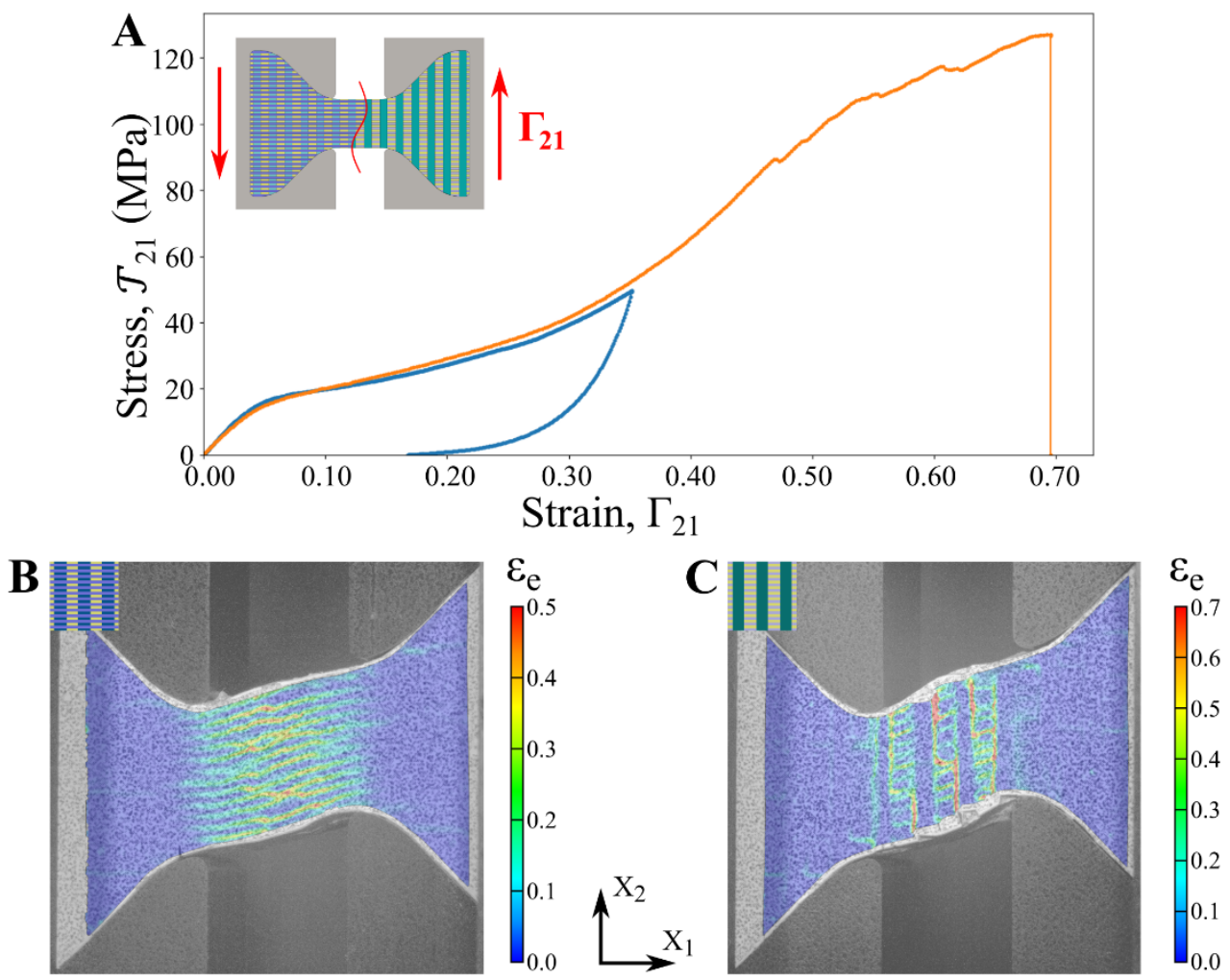

Figure 7: The shear response in the $Z X$ orientation. (A) The measured $\mathcal{T}_{21}$ versus $\Gamma_{21}$ responses from two separate measurements including one for a specimen taken to complete fracture. Measurements of the distribution of the effective strain $\varepsilon_{e}$ on (B) the $X$-section and (C) the $Z$-section of the specimens loaded to $\Gamma_{21} \approx 30 \%$. The sketches in the insets use the colour scheme from Fig. 1.

Similar to the $X Z$ orientation, there was considerable strain recovery for specimens loaded to strains below ultimate failure strain. For example, a specimen loaded to $\Gamma_{21} \approx 32 \%$ recovered $\sim 50 \%$ of the applied strain upon unloading; see Fig. 7A. Moreover, the deformation and recovery mechanisms are similar for the two orientations, i.e. shear deformation in the $Z X$ orientation is accompanied by elastic tensile straining of the $X$-tows which is a major cause of the hardening and recovery after unloading. Ultimate failure results from fracture of the $X$ tows. These tows have a higher volume fraction of fibres compared to the $Z$-tows resulting in the $Z X$ orientation specimens having a higher ultimate strength. 


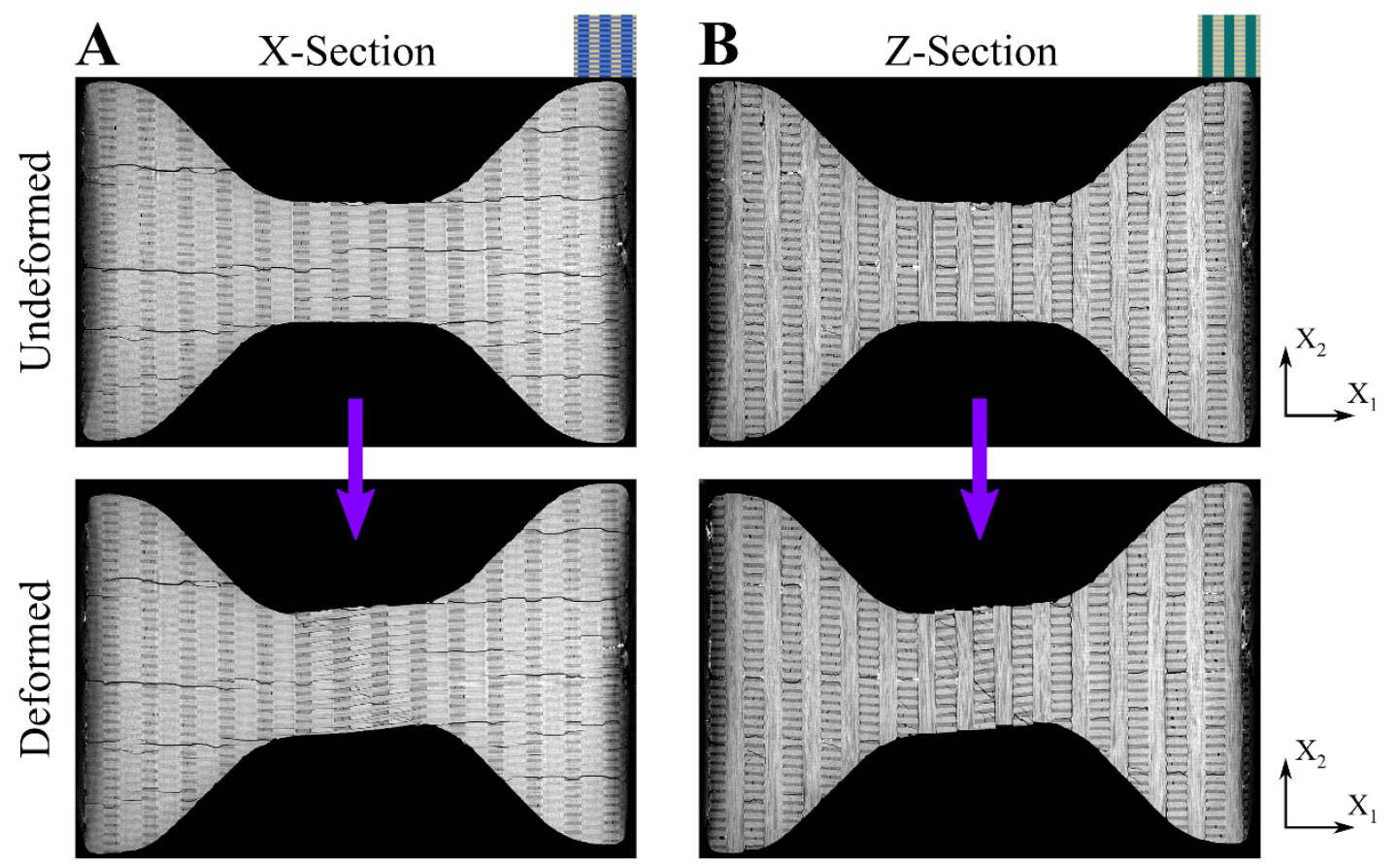

Figure 8: X-ray tomographic images of the (A) $X$-sections and (B) $Z$-sections of the specimen in the $Z X$ orientation. Images are shown of both the undeformed specimens and the specimen unloaded after application of a shear strain $\Gamma_{21} \approx 35 \%$. The sketches indicate the section of the specimen using the colour scheme from Fig. 1.

\subsubsection{Deformation modes and $X$-ray tomography observations}

$\mathrm{X}$-ray CT images of the $X$ and $Z$-sections of the specimen in the $Z X$ orientation prior to testing and after unloading from an applied shear strain $\Gamma_{21} \approx 35 \%$ are included in Fig. 8. Prior to testing, the specimens have a similar defect landscape to that of the $X Z$ orientation specimens, albeit rotated by $90^{\circ}$. After loading, cracks in the $X_{1}$-direction between the $X$ - and $Y$-tows tended to close (Fig. 8A). In the $Z$-section of the specimen, there were some observable shear cracks that pass through the $Y$-tows and the matrix pockets (Fig. 8B). To quantify this deformation, we include spatial distributions of $\varepsilon_{e}$ (as measured via DIC) for the $X$ and $Z$ sections in Figs. 7B and 7C, respectively. These distributions are shown at an applied $\Gamma_{21} \approx$ $30 \%$. The DIC measurements are consistent with the XCT images and show deformations in areas of the specimen where microcracking occurs. Large strain concentrations are also seen along the $Z$-tows in the $Z$-section. This heterogeneous deformation is primarily a result of the mismatch in properties between the tows and the intervening matrix pockets.

X-ray CT images of the $X$ and $Z$-sections of the failed specimens are shown in Figs. 6C and $6 \mathrm{D}$, respectively. The final rupture of the $X$-tows occurred near the edges of the gauge section and were again accompanied by considerable tow pull-out and the associated zig-zag fracture surfaces. The $Z$-tows remained relatively undamaged throughout the deformation, and the $Y$ tows displayed some shear damage as visible in the $Z$-section of the specimen (Fig. 6D). Notably, some of the $X$ - and $Y$-tows at the edge of the gauge section delaminated and completely detached from the intersecting $Z$-tows, leading to fracture on disconnected planes as seen in Fig. 6C. 

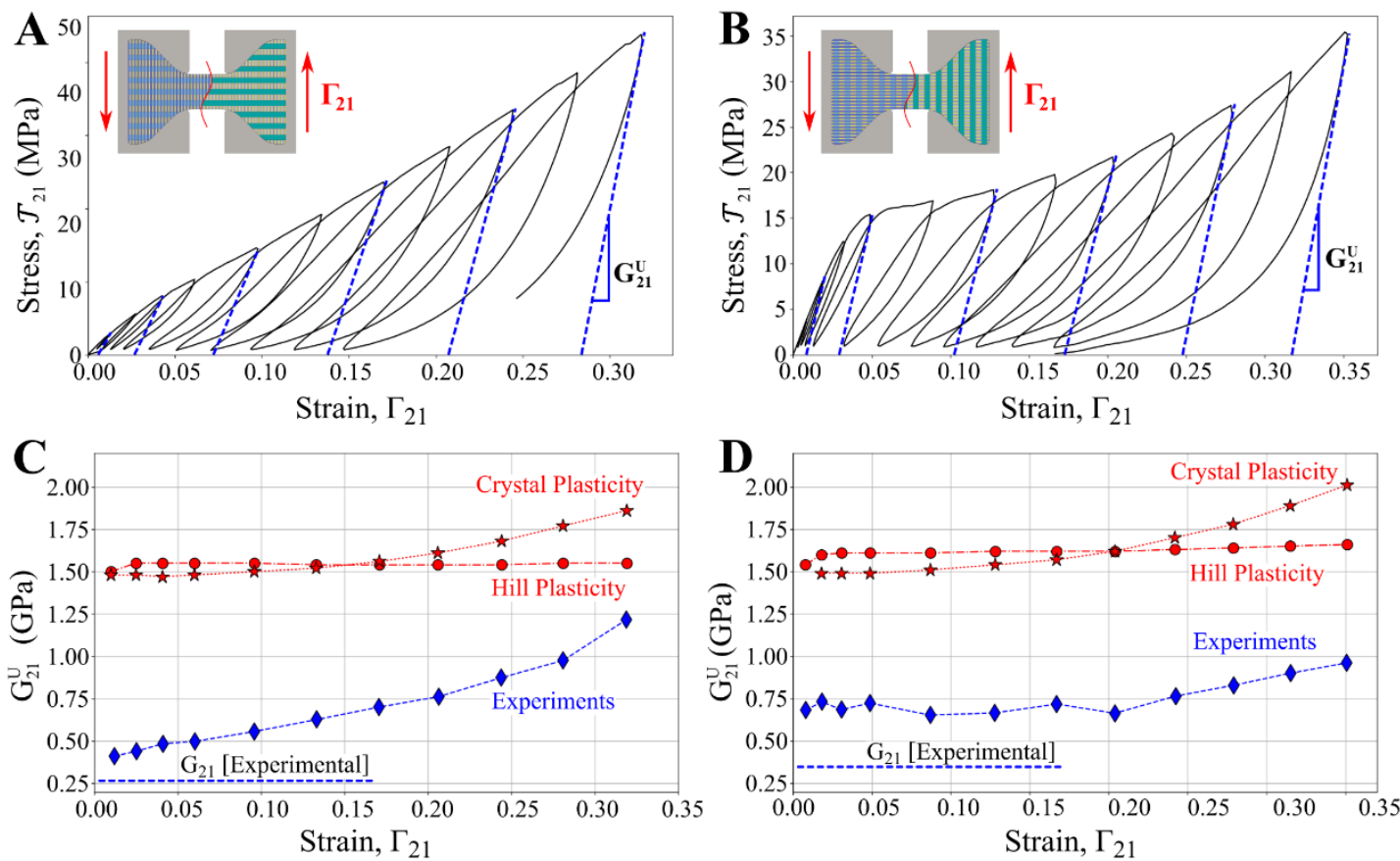

Figure 9: The measured loading/unloading responses of the specimens in the (A) $X Z$ and (B) $Z X$ orientations. The unloading stiffness is shown for selected unloading cycles. The measurements and predictions of the evolutions of the unloading stiffnesses $G_{21}^{U}$ for the (C) $X Z$ and (D) $Z X$ orientation samples with shear strain $\Gamma_{21}$. Predictions are shown for both the Hill and crystal plasticity models for the tows. The measured initial loading stiffnesses are also indicated as $G_{21}$ for comparison purposes.

\subsection{Effect of damage accumulation and the loading/unloading response}

Damage accumulation in traditional CFRP composites is often characterised by the loss of stiffness of the composite (Lafarie-Frenot and Touchard, 1994; Tan and Falzon, 2016). Here we attempt a similar characterisation to highlight the differences between traditional composites and these 3D noobed composites. The measured loading/unloading responses of the specimens in the $X Z$ and $Z X$ orientations are included in Figs. 9A and 9B, respectively along with the unloading stiffnesses inferred from selected loading/unloading cycles. Unloading in both orientations results in significant strain recovery with an associated hysteresis akin to the Bauschinger effect in metals. This recovery and hysteresis are associated with reverse plasticity arising from elastic tensile straining of the tows as will be discussed in the context of the numerical simulations in Section 5.

The evolution of the unloading stiffnesses $G_{21}^{U}$ with shear deformation $\Gamma_{21}$ for the $X Z$ and $Z X$ orientation specimens is plotted in Figs. 9C and 9D, respectively. The unloading stiffness for the $X Z$ specimens monotonically increases with increasing $\Gamma_{21}$ over the entire range of shear strains investigated here, while unloading stiffness for the $Z X$ specimens remains relatively constant for $\Gamma_{12} \leq 20 \%$ but increases thereafter. This increase in stiffness with deformation is contrary to most reported measurements for traditional CFRP composites, which typically show a reduction in stiffness with increasing deformation due to a variety of cracking and delamination damage mechanisms. In fact, the stiffness reduction in CFRP composites is regularly used to quantify their damage level. These 3D noobed composites have a markedly different response due to two interconnected reasons:

(i) The 3D architecture of these composites inhibits the traditional damage modes such as delamination (recall XCT images) and helps bind the tows together to retain structural integrity up to very large imposed shear strains. 
(ii) The large shear strain results in significant tow rotation, notably of the $X_{1}$-direction tows. This reorientation causes axial stretching of tows and contributes to the increase in the specimen shear stiffness.

It is worth mentioning here that we anticipate stiffening due to tow reorientation to also occur in $2 \mathrm{D}$ composites. However, damage mechanisms often cause a knock-down the constituent material properties of such composites, negating any stiffening at large strains due to reorientation effects. Thus, we argue that it is the damage inhibition in these noobed 3D composites that leads to the observed anomalous evolution of the unloading stiffness.

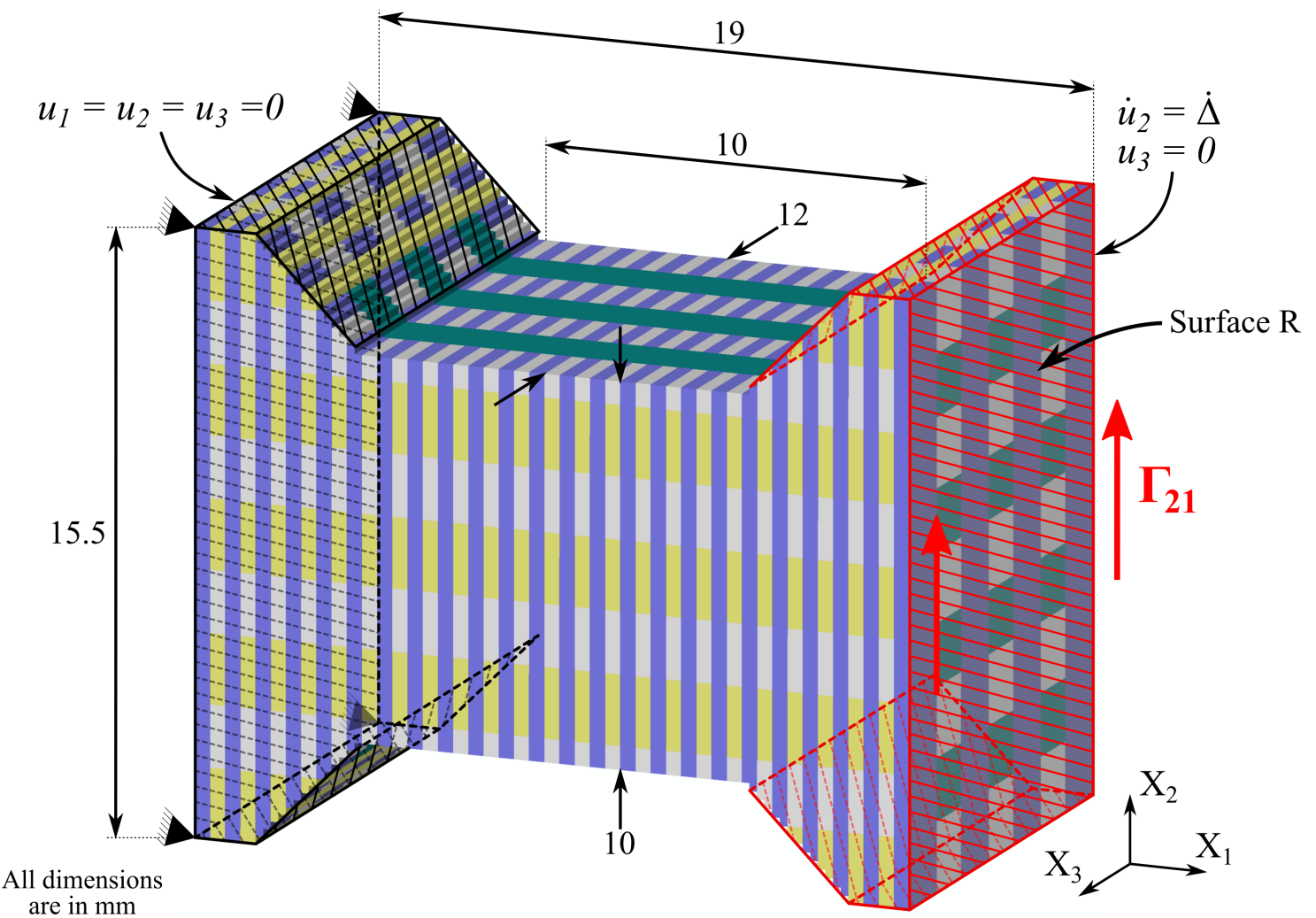

Figure 10: Sketch of the truncated dogbone specimen the used in the numerical model along with the applied boundary conditions. The hatched regions indicate the "grip" regions of the model, i.e. regions of the specimen that are within the grips and on which displacement boundary conditions are been applied in the FE calculations. The sketch shows the specimen in the $X Z$ orientation with leading specimen dimensions marked.

\section{Numerical modelling of 3D noobed composites}

The architecture of 3D fibre composites is intrinsically complex with multiple materials and length scales that govern their global behaviour. The aim here is not to explicitly model all the microstructural features and defects of these composites but to attempt to understand the deformation and failure mechanisms via the simplest homogenised models that capture the critical experimental observations. Das et al. (2018) employed a homogenised model wherein only the $Z$-tows of the 3D noobed composites were explicitly modelled using a Hill anisotropic plasticity model (Hill, 1948), with the remainder of the composite also modelled using a Hill anisotropic plasticity model but with properties derived by homogenising the remaining tows and matrix pockets. However, numerical deficiencies were observed with this level of simplification, including an inability of the model to capture the responses under different stress states. Here we shall employ a more complex approach whereby all the tows and matrix pockets are explicitly modelled as homogenised continua. The emphasis will be on detailing 
the model features required to capture the large strain composite response with sufficient fidelity.

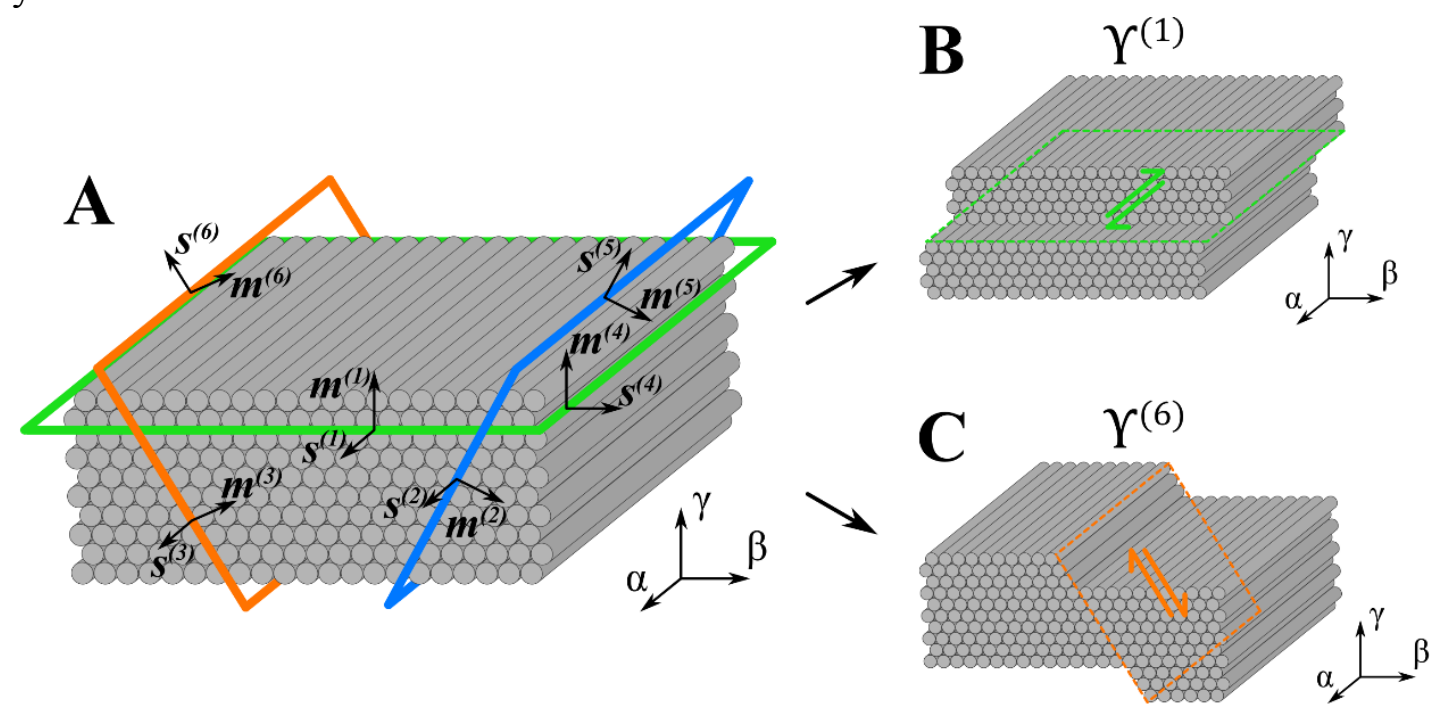

Figure 11: Sketches to illustrate the deformation of a tow as modelled via a crystal plasticity-based model. (A) The local co-ordinate system $(\alpha, \beta, \gamma)$ of a single tow and the six slip systems $\left(s_{i}^{(\varpi)}, m_{i}^{(\varpi)}\right)$ via which the tows deform plastically. (B) Tow deformation subjected to a simple shear strain resulting in slip on slip system $\varpi=1$. (C) Tow deformation due to slip on slip system $\varpi=6$.

\subsection{Description of numerical model}

We modelled a truncated dogbone specimen as sketched in Fig. 10; the ends of the dogbone deep within the grips excluded from the model to reduce the computational cost. The finite strain finite element calculations were performed using the commercial package ABAQUS/Explicit (Dassault Systèmes, France). Shear loading was imposed by applying a constant displacement rate $\dot{u}_{2}=\dot{\Delta}$ (with $u_{3}=0$ ) to all surface nodes on the right "grip" region of the specimen (labelled ' $R$ ' in Fig. 10 and indicated by the hatched area) while fully constraining the displacements $u_{i}$ of all surface nodes on the opposite grip region of the specimen. No constraint was specified on displacements $u_{1}$ on grip region $R$, implying that the tractions $T_{1}\left(x_{i}\right)=0$ on that surface. This is consistent with the experimental boundary conditions that ensure that the axial force $P_{1}=0$.

All tows and matrix pockets within the specimen were modelled explicitly. The microstructure was assumed to be perfectly regular, and imperfections such as the tow waviness and interfacial cracks were not considered. Thus, the sizes of the $X, Y$ and $Z$-tows and the matrix pockets were taken to be equal to those specified in Fig. $1 \mathrm{~B}$, i.e. equal to an average value inferred from the $\mathrm{XCT}$ images. The dogbone with this microstructure was discretised using rectangular hexahedral elements (C3D8 in the ABAQUS notation) such that at-least four finite elements were present across the smallest dimension of every tow and matrix pocket. This resulted in approximately 2 million C3D8 elements in the model with 8 million degrees of freedom.

\subsection{Material properties}

The 3D composite comprised NM FW 3070 epoxy matrix and T700S carbon fibres. To minimize the complexity of the model, both the matrix and fibres are assumed to be isotropic with Young's moduli $E_{m}$ and $E_{f}$, respectively and Poisson's ratios $v_{m}$ and $v_{f}$, respectively. The fibres are elastic/brittle with a tensile failure strength $\Sigma_{f}$, while the matrix is taken to be perfectly plastic with a tensile yield strength $\Sigma_{m}$. These properties, as specified by the manufacturer, are all listed in Table 1. We use these properties in our calculation of the 
homogenized properties of the constituent materials, i.e. the individual tows and the matrix pockets as will be clarified subsequently.

Table 1: Elastic and plastic properties of the fibre and matrix.

\begin{tabular}{l|c|l|l|c|l}
\multicolumn{2}{l|}{ T700S Carbon Fibre } & \multicolumn{3}{l}{ NM FW 3070 Epoxy } \\
\hline $\boldsymbol{E}_{\boldsymbol{f}}(\mathrm{GPa})$ & $v_{f}$ & $\Sigma_{f}(\mathrm{MPa})$ & $E_{m}(\mathrm{GPa})$ & $v_{m}$ & $\Sigma_{m}(\mathrm{MPa})$ \\
\hline 230 & 0.28 & 4,900 & 2.70 & 0.3 & 17
\end{tabular}

\subsubsection{Elastic properties}

The individual tows are unidirectional fibre composites and are modelled as transversely isotropic continua with the fibre direction normal to the plane of isotropy. To specify these material properties, we define a Cartesian co-ordinate system $(\alpha, \beta, \gamma)$ local to each tow such that $\alpha$ is along the fibre direction with $(\beta, \gamma)$ forming the isotropic plane perpendicular to the fibres (Fig. 11A). In describing the constitutive models for the tows, we use the local coordinate system $(\alpha, \beta, \gamma)$ with all tensor quantities (e.g. $\left.\sigma_{i j}\right)$ defined with respect to this basis. The relation between the elastic strains $\varepsilon_{i j}^{*}$ and the Cauchy stress $\sigma_{i j}$ is then written in terms of the compliance tensor $C_{i j k l}$ as $\varepsilon_{i j}^{*}=C_{i j k l} \sigma_{k l}$, and succinctly using Voigt notation as

$$
\left(\begin{array}{c}
\varepsilon_{\alpha \alpha}^{*} \\
\varepsilon_{\beta \beta}^{*} \\
\varepsilon_{\gamma \gamma}^{*} \\
\varepsilon_{\beta \gamma}^{*} \\
\varepsilon_{\alpha \gamma}^{*} \\
\varepsilon_{\alpha \beta}^{*}
\end{array}\right)=\left(\begin{array}{cccccc}
\frac{1}{E_{\alpha}} & -\frac{v_{\alpha \beta}}{E_{\alpha}} & -\frac{v_{\alpha \beta}}{E_{\alpha}} & 0 & 0 & 0 \\
-\frac{v_{\alpha \beta}}{E_{\alpha}} & \frac{1}{E_{\beta}} & -\frac{v_{\beta \gamma}}{E_{\beta}} & 0 & 0 & 0 \\
-\frac{v_{\alpha \beta}}{E_{\alpha}} & -\frac{v_{\beta \gamma}}{E_{\beta}} & \frac{1}{E_{\beta}} & 0 & 0 & 0 \\
0 & 0 & 0 & \frac{1+v_{\beta \gamma}}{E_{\beta}} & 0 & 0 \\
0 & 0 & 0 & 0 & \frac{1}{2 G_{\alpha \beta}} & 0 \\
0 & 0 & 0 & 0 & 0 & \frac{1}{2 G_{\alpha \beta}}
\end{array}\right)\left(\begin{array}{c}
\sigma_{\alpha \alpha} \\
\sigma_{\beta \beta} \\
\sigma_{\gamma \gamma} \\
\sigma_{\beta \gamma} \\
\sigma_{\alpha \gamma} \\
\sigma_{\alpha \beta}
\end{array}\right) .
$$

The transversely isotropic elastic constants of the $X / Y$ and $Z$-tows are calculated from the matrix and fibre properties using the homogenisation analysis detailed Appendix B, and the resulting tow elastic constants are listed in Table 2. Further, we assume the matrix to be isotropic linear elastic with elastic constants given in Table 1. It now remains to specify in inelastic behaviour of the matrix pockets and tows.

Table 2: Elastic properties of the $X, Y$, and $Z$-direction tows.

\begin{tabular}{c|l|l|l|r|c} 
Tow & $E_{\alpha}(\mathrm{GPa})$ & $E_{\beta}(\mathrm{GPa})$ & $G_{\alpha \beta}(\mathrm{GPa})$ & $v_{\alpha \beta}$ & $v_{\beta \gamma}$ \\
\hline$X / Y$ & 144 & 16.2 & 4.79 & 0.288 & 0.451 \\
\hline$Z$ & 93.6 & 14.9 & 4.27 & 0.292 & 0.460
\end{tabular}

\subsubsection{Choice of plasticity models for fibre tows}

The tows are anisotropic continua and their plastic response is most commonly modelled using quadratic yield criteria (Vogler et al., 2013). The Hill orthotropic plasticity model (Hill, 1948) is one such simple and convenient criterion that is regularly used for modelling composites 
(Ansar et al., 2011). However, as discussed in Section 1, the most commonly used variant of the Hill plasticity model rotates the material substructure with the total deformation gradient (Beissel, 2014; Choi et al., 2018; Hasanyan and Waas, 2018). This variant thus neglects plastic spin and thereby will be unable to accurately capture the evolution of the tow substructure at finite strains. This texture evolution within the tows is expected to be important in these high ductility 3D composites and constitutive models that do not include plastic spin are therefore expected to have poor predictive capability for the response of 3D composites. We show this explicitly in Appendix C, where we provide the formulation of the Hill model absent plastic spin (for the sake of brevity we shall subsequently refer to it simply as the Hill model) and include comparisons with experimental measurements.

The two key assumptions of our Hill plasticity model that result in its poor predictive capability for 3D composites are: (i) the material symmetries remain unchanged with plastic deformation and (ii) the kinematics of the material substructure (i.e. the fibres in this case) are identical to that of the continuum. While (i) implies that a quadratic plastic potential of the form (C.3) can describe the continuing yielding of the tow, the implication of (ii) is that the rotation of the local tow level co-ordinate system $(\alpha, \beta, \gamma)$ with respect to the global co-ordinate system $X_{i}$ is equal to the spin component of the total material deformation gradient $F_{i j}$. To illustrate this, consider the simple shear $\Gamma_{\alpha \gamma}$ of a tow with a rigid-plastic matrix (Fig. 11B). While the material rotation in this case is $\Gamma_{\alpha \gamma} / 2$, the fibre rotation is zero. However, the finite strain Hill plasticity model will rotate the tow coordinates $(\alpha, \beta, \gamma)$ by $\Gamma_{\alpha \gamma} / 2$ even though in this case the fibres would not have rotated. This results in errors in the prediction of the large strain response of 3D composites as seen in Appendix C and further discussed in Section 5.

A more accurate description of the anisotropic plastic deformation can be developed by introducing the notion of plastic spin within the Hill plasticity model; see for example Dafalias (1984) and Aravas, (1994). This typically involves specification of additional tensorial constitutive rules for plastic spin, although the assumption that the material symmetries are invariant to plastic deformation typically remains. Rather than following this route, here we take the view that given the texture/substructure of the tows, it is natural to describe the plastic deformation of tows via a crystal plasticity-based approach. Such an approach was followed recently by Liu et al. (2018) for describing the large strain deformation of ultra-high molecular weight polyethylene fibre composites. We now proceed to summarise such a crystal plasticity model that includes pressure dependent yield. Here, pressure dependency is introduced both because the polymer matrix is pressure dependent (Rabinowitz et al., 1970; Ward, 1971) and because tows with a high volume fraction of fibres are reminiscent of a granular medium and hence are expected to have a pressure dependent yield response akin to granular materials.

\subsubsection{Crystal plasticity-based model for tows}

In order to overcome the shortcomings of the Hill model absent plastic spin, here we propose a crystal plasticity model for the individual tows that inherently captures their internal texture evolution. The plastic deformation of a tow is similar to a crystal that deforms by shearing along slip planes due to the motion of dislocations (see for example Figs. 11B and 11C). In fact, such localised shear deformation is common in unidirectional composites as reported by González and LLorca (2007). Thus, we proceed to develop a constitutive model for tows motivated by crystal plasticity descriptions for metal single crystals (Asaro, 1983; Hill and Rice, 1972). Following the usual notions in crystal plasticity, we define a lattice labelled by the

fibre direction. The material is presumed to flow through the lattice due to shearing of the matrix and then the lattice with the embedded material undergoes elastic deformations and rigid 
body rotations. Using Cartesian tensor notation, the material deformation gradient $F_{i j}$ is written as a multiplicative decomposition of the elastic and plastic deformations characterised by $F_{i j}^{*}$ and $F_{i j}^{p}$, respectively such that

$$
F_{i j}=F_{i k}^{*} F_{k j}^{p} \text {. }
$$

Thus, there exists an intermediate configuration arising from the pure plastic deformation $F_{i j}^{p}$ of the material. The deformations embodied in $F_{i j}^{p}$ are described in terms of shearing along crystallographic slip systems set by the fibre direction. A given slip system $(\varpi)$ is specified by vectors $s_{i}^{(\varpi)}$ and $m_{i}^{(\varpi)}$, where $s_{i}^{(\varpi)}$ specifies the slip direction and $m_{i}^{(\varpi)}$ is the slip plane normal in the undeformed configuration. These vectors convect with the lattice so that in the deformed state they become

$$
s_{i}^{*(\varpi)}=F_{i j}^{*} s_{j}^{(\varpi)} \quad \text { and } \quad m_{i}^{*(\varpi)}=m_{j}^{(\varpi)}\left(F_{j i}^{*}\right)^{-1} .
$$

For a tow whose $(\alpha, \beta, \gamma)$ coordinate system is aligned with the global $\left(X_{1}, X_{2}, X_{3}\right)$ coordinates, we consider $N=6$ slip systems as listed in Table 3 and illustrated in Fig. 11A. The slip systems $\varpi=1-3$ involve longitudinal shearing in the $\alpha$ (fibre) direction, while the $\varpi=4-6$ systems involve transverse shearing in the $(\beta, \gamma)$ plane with no shear component along the $\alpha$ direction. These slip systems represent the minimum set of slip systems to accommodate all possible modes of deformation that do not involve fibre deformation, with systems $\varpi=4-6$ resulting in an approximately isotropic plastic response in the $(\beta, \gamma)$ plane consistent with the assumed transversely isotropic behaviour. Longitudinal and transverse shear along with their corresponding slip systems are illustrated in Figs. 11B and 11C, respectively. We emphasize that the precise orientations of these slip systems will have only a minor effect on the overall numerical results as perpendicular to the fibre directions there are sufficient slip systems to accommodate any imposed strain state via purely plastic deformations.

The plastic component of the deformation gradient and the corresponding velocity gradient $L_{i j}^{p}$ associated with plastic shearing on the slip systems then follow as

$$
L_{i j}^{p}=F_{i k}^{*} \dot{F}_{k l}^{p}\left(F_{l m}^{p}\right)^{-1}\left(F_{m j}^{*}\right)^{-1}=\sum_{\varpi=1}^{N} \dot{\Upsilon}^{(\varpi)} s_{i}^{*(\varpi)} m_{j}^{*(\varpi)},
$$

where $\dot{\Upsilon}^{(\varpi)}$ is the slip rate on slip system $(\varpi)$ while the total velocity gradient is $L_{i j}=\dot{F}_{i k}\left(F_{k j}\right)^{-1}$ with $L_{i j}^{*} \equiv L_{i j}-L_{i j}^{p}$ being the elastic component of the velocity gradient. The corresponding symmetric parts of the elastic and plastic velocity gradients representing the stretching (straining) then follow as

$$
\dot{\varepsilon}_{i j}^{*}=\left(L_{i j}^{*}+L_{j i}^{*}\right) / 2 \text { and } \dot{\varepsilon}_{i j}^{p}=\left(L_{i j}^{p}+L_{j i}^{p}\right) / 2 \text {, }
$$

respectively while elastic and plastic spin rates are

$$
\dot{w}_{i j}^{*}=\left(L_{i j}^{*}-L_{j i}^{*}\right) / 2 \text { and } \dot{w}_{i j}^{p}=\left(L_{i j}^{p}-L_{j i}^{p}\right) / 2 \text {, }
$$

respectively. The hypoelastic relation between the elastic strain rate and stress rates is then given by a rate form of (4.1) such that $\dot{\varepsilon}_{i j}^{*}=C_{i j k l}^{\prime}{ }^{\nabla}{ }_{k l}$, where

$$
{ }_{\tau_{i j}}^{\nabla} \equiv \dot{\tau}_{i j}-\dot{w}_{i k}^{*} \tau_{k j}+\tau_{i k} \dot{w}_{k j}^{*}
$$


is the Jaumann rate of the Kirchhoff stress $\tau_{i j}=J \sigma_{i j}$ with respect to axes that rotate with the lattice. Here with $J=\operatorname{det}\left(F_{i j}^{*}\right)$ and $C_{i j k l}^{\prime}=F_{i n}^{*} F_{j m}^{*} F_{k p}^{*} F_{l q}^{*} C_{n m p q}$ are components of the elastic compliance tensor in the deformed configuration.

It now remains to specify the constitutive relations for the plastic slip rate $\dot{\Upsilon}^{(\varpi)}$. Significant numerical difficulties are associated with determining the active slip systems and the amount of slip on each of these systems if a rate independent model is employed for $\dot{\Upsilon}^{(\varpi)}$. Thus, Asaro and Needleman (1985) proposed a simple rate dependent crystal plasticity formulation that provides a good approximation to the rate independent limit and circumvents these numerical issues. We employ their methodology here. Plastic deformation due to shearing on each slip system $(\varpi)$ depends on the resolved shear stress

$$
\tau^{(\varpi)}=J m_{i}^{*(\varpi)} \sigma_{i j} s_{j}^{*(\varpi)} .
$$

However, unlike metallic crystals, plastic flow in the matrix infused carbon fibre tows is pressure dependent as discussed above. Here we characterise this pressure dependency via a friction coefficient $\mu$ and define a slip system shear strength

$$
g^{(\varpi)}= \begin{cases}\tau_{y}+\mu p & p \geq 0 \\ \tau_{y} & p<0\end{cases}
$$

where pressure $p$ is a measure of the pressure absent the fibre stresses. This pressure is thus defined in terms of the material stresses in the intermediate configuration as

$$
p \equiv-\frac{1}{2}\left(\Sigma_{\beta \beta}+\Sigma_{\gamma \gamma}\right)
$$

with $\Sigma_{i j}=\operatorname{det}\left(F_{i j}^{*}\right)\left(F_{i k}^{*}\right)^{-1} \sigma_{k l}\left(F_{j l}^{*}\right)^{-1}$. Then following Asaro and Needleman (1985), the shear rate $\dot{\Upsilon}^{(\varpi)}$ is specified by a rate dependent law as

$$
\dot{\Upsilon}^{(\varpi)}=\dot{\Upsilon}_{0}\left(\frac{\left|\tau^{(\varpi)}\right|}{g^{(\varpi)}}\right)^{1 / m} \operatorname{sgn}\left[\tau^{(\varpi)}\right],
$$

where $\dot{\Upsilon}_{0}$ is a reference strain rate and $m$ is a rate sensitivity exponent such that the rate independent limit is retrieved as $m \rightarrow 0$. In the calculations presented here, we set $\tau_{y}=\tau_{m}=$ $10 \mathrm{MPa}$, i.e. equal to the shear strength of the matrix. The rate sensitivity exponent is chosen to be $m=0.2$, which is sufficient to avoid numerical instabilities but yet give a reasonable approximation to the rate independent limit. Finally, $\dot{\Upsilon}_{0}$ is set equal to the imposed loading rate for the model. Unless otherwise specified, results are presented for a pressure sensitivity of $\mu=0.2$, which is equal to that of polymers that are similar to the matrix used here (Rabinowitz et al., 1970). We also present some predictions with no pressure dependent yield (i.e. $\mu=0$ ) to quantify the effect of the pressure sensitivity of the tow response.

\subsubsection{Inelastic model for the matrix in the matrix pockets}

In keeping with the understanding that the yield behaviour of the polymer matrix is pressure dependent (Rabinowitz et al., 1970; Sauer, 1977), we model the matrix pockets via an isotropic elastic-plastic non-associative Drucker-Prager material model (Drucker and Prager, 1952). The total strain rate is given by additive decomposition, i.e. $\dot{\varepsilon}_{i j}=\dot{\varepsilon}_{i j}^{*}+\dot{\varepsilon}_{i j}^{p}$. Consistent with a wide body of experimental polymer yield data (Ward, 1971), we assume that plastic straining is incompressible so that the plastic strain rate $\dot{\varepsilon}_{i j}^{p}$ under active yield conditions given in terms of the plastic multiplier $\dot{\lambda}$ via

$$
\dot{\varepsilon}_{i j}^{p}=\dot{\lambda} \frac{\partial \sigma_{e}}{\partial \sigma_{i j}}
$$


where $\sigma_{e} \equiv \sqrt{(3 / 2) s_{i j} s_{i j}}$ is the von-Mises stress in terms of the deviatoric stress $s_{i j}$. The yield criterion is specified via the surface

$$
\Phi \equiv \sigma_{e}-\mu_{m} p-\Sigma_{m}\left(1-\frac{\mu_{m}}{3}\right)
$$

where $p \equiv-\sigma_{k k} / 3$ is the hydrostatic pressure and continued plastic straining occurring when $\Phi=0$. The matrix strength $\Sigma_{m}$ is listed in Table 1. In all the numerical results presented here, we set $\mu_{m}=0.2$, irrespective of the material model and pressure sensitivity used for the tows.

Table 3: The six slip systems in the tows as specified by $\left(s_{i}^{(\varpi)}, m_{i}^{(\varpi)}\right)$. The unit vectors are denoted as $(a, b, c)$ where $a, b, c$ are components in the $\alpha, \beta$ and $\gamma$-directions, respectively. These slip systems are sketched in Fig. 11.

\begin{tabular}{c|c|c|c}
\multicolumn{2}{c|}{ Slip Direction } & \multicolumn{2}{c}{ Slip Plane Normal } \\
\hline $\boldsymbol{s}^{(\mathbf{1})}$ & $(1,0,0)$ & $m^{(1)}$ & $(0,0,1)$ \\
$\boldsymbol{s}^{(2)}$ & $(1,0,0)$ & $m^{(2)}$ & $(0, \sqrt{3} / 2,-1 / 2)$ \\
$\boldsymbol{s}^{(3)}$ & $(1,0,0)$ & $m^{(3)}$ & $(0, \sqrt{3} / 2,1 / 2)$ \\
$\boldsymbol{s}^{(\mathbf{4})}$ & $(0,1,0)$ & $m^{(4)}$ & $(0,0,1)$ \\
$\boldsymbol{S}^{(\mathbf{5})}$ & $(0,1 / 2, \sqrt{3} / 2)$ & $m^{(5)}$ & $(0, \sqrt{3} / 2,-1 / 2)$ \\
$\boldsymbol{s}^{(\mathbf{6})}$ & $(0,-1 / 2, \sqrt{3} / 2)$ & $m^{(6)}$ & $(0, \sqrt{3} / 2,1 / 2)$
\end{tabular}

\section{Comparison between measurements and numerical predictions}

We now proceed to discuss comparisons between the experimental measurements and numerical predictions for the shear response of the 3D composites. The discussion primarily focuses on the large strain predictions of the crystal plasticity model, and includes some references to differences with the Hill model predictions given in Appendix C. Moreover, in order to interpret some of the key predictions of the crystal plasticity model and highlight the role of plastic spin, we also include here the Hill predictions for the spatial distributions of stresses and strains within the specimen alongside those from the crystal plasticity model.

Prior to describing the results of the model, it is important to comment on the material orientations within the tows. The fibre direction $(\alpha)$ of the tows is defined unambiguously within the specimens, i.e. it is aligned parallel to the longitudinal direction of the tow such that $\alpha$ is parallel to $X_{3}$ for the $Y$-tows while $\alpha$ is parallel to $X_{1}$ for the $Z$-tows in the $X Z$-orientation specimens and so on. However, the $(\beta, \gamma)$ directions in the tows are uncertain and in fact could vary within the specimen due to twist of the tow. While the elastic tow model is transversely isotropic with the $\alpha$-direction perpendicular to the plane of isotropy, the plastic properties are in general not isotropic in that plane. Numerical studies demonstrated that a $30^{\circ}$ rotation of the tow about the $\alpha$-direction led to less than a 4\% change in the shear stress at a given applied $\Gamma_{21}$. This suggests that the orientation of the tow about the $\alpha$-direction has a negligible effect on the composite properties investigated here. This result might have been expected as there are at-least three independent slip systems to accommodate plastic strain in the plane orthogonal to the $\alpha$-direction, meaning the plastic response of the tows in that plane is approximately isotropic. Therefore, all computations presented subsequently use the following mappings of the initial tow directions with the global coordinate system: $(\alpha, \beta, \gamma) \rightarrow\left(X_{1}, X_{2}, X_{3}\right)$ for tows in the $X_{1}$ direction, $(\alpha, \beta, \gamma) \rightarrow\left(X_{2},-X_{1}, X_{3}\right)$ for tows in the $X_{2}$ direction, and $(\alpha, \beta, \gamma)$ $\rightarrow\left(X_{3}, X_{2},-X_{1}\right)$ for tows in the $X_{3}$ direction. In the following, all tensor quantities (e.g. 
deformation gradients $F_{i j}^{p}$, stresses $\sigma_{i j}$, plastic strains $\varepsilon_{i j}^{p}$ etc.) are shown using global coordinate system $X_{i}$ as the basis rather than the local system $(\alpha, \beta, \gamma)$ used in describing the constitutive models in Section 4.

\subsection{Comparisons with model predictions}

The crystal plasticity model predictions, both with and without pressure dependent yield, are shown against the experimental measurements in Figs. 12A and $12 \mathrm{~B}$ for the $X Z$ and $Z X$ orientations, respectively. The model overpredicts the initial shear modulus in both orientations, but it predicts the initial yield for both orientations with reasonable accuracy. The shear modulus overprediction is expected given that the elastic properties used in the model neglect imperfections (Section 4.2). Imperfections like microcracking (Tao and Sun, 1996) and fibre and tow level waviness (Petriccione et al., 2012) are known to have a detrimental effect on the stiffness, sometimes reducing it by nearly an order of magnitude. Knockdown factors to account for these imperfections could be included to bring the predicted stiffnesses in better alignment with the measurements. However, given that the focus of the numerical investigation was to gain an understanding of the large-strain deformation mechanisms, such a fitting exercise was not carried out.

The post-yield behaviour of the crystal plasticity model for both composite orientations is in good agreement with the measurements, particularly for the model with pressure dependence $(\mu=0.2)$. Switching-off pressure dependent yield $(\mu=0)$ leads to a reduced but nonnegligible hardening response, demonstrating that the hardening shear behaviour is not solely the result of pressure dependent yield.
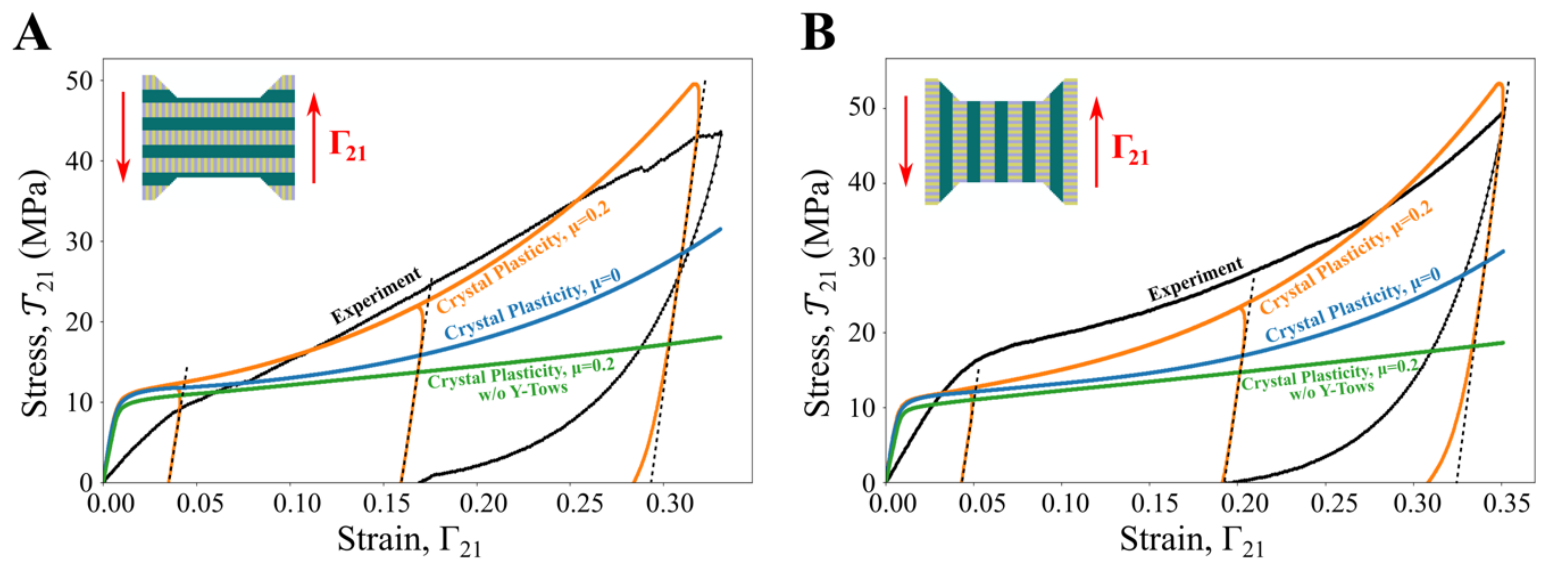

Figure 12: Comparisons between measurements and predictions using the crystal plasticity model for the tows of the shear responses in the (A) $X Z$ and (B) $Z X$ orientations. The crystal plasticity predictions are shown for the reference tow pressure sensitivity $\mu=0.2$ and no pressure sensitivity with $\mu=0$. Predictions are also shown with the $Y$-tows replaced by matrix material (w/o $Y$-tows). In addition, predictions are shown for unloading from selected values of the applied shear strains $\Gamma_{21}$ for the reference case with $\mu=0.2$. The specimen sketches use the colour scheme from Fig. 1.

The ability of the crystal plasticity model to capture the hardening is also reflected in the unloading stiffnesses predictions, where the model correctly predicts a monotonic increase in $G_{21}^{U}$ with increasing $\Gamma_{21}$ (Figs. 9C and 9D). In addition, the crystal plasticity model predicts some reverse plasticity during unloading at large applied $\Gamma_{21}$ (Fig. 12), although the degree of reverse plasticity is less than that in the experiments. This is as contrary to the Hill plasticity model, which predicts no reverse plasticity (Fig. C1). Overall, the hardening response, increase in $G_{21}^{U}$ with increasing shear strain and the reverse plasticity are all connected to the elastic straining of the tows that occurs due to the inhomogeneous plastic strains in the composite. To 
illustrate this, we include in Fig. 13A predictions of the distribution of plastic strain $\varepsilon_{12}^{p}$ in the $X$ - and $Z$-sections of the $X Z$ orientation specimen at an applied $\Gamma_{21}=40 \%$. The plastic strain distributions are highly spatially inhomogeneous, with large plastic strain concentrations arising at the interfaces of the tows. However, compatibility requires the total strains in the gauge section to be approximately spatially uniform, so the inhomogeneous plastic strains imply plastic incompatibility and large elastic strains arise to enforce strain compatibility. This plastic incompatibility manifests predominantly at large applied strains and is due to plastic spin and texture evolution within the tows as will be discussed in Section 5.2. By comparison, results from the Hill plasticity model in Fig. 13B show a relatively homogeneous plastic strain distribution, and correspondingly very little build-up of elastic strain or hydrostatic pressure. This is discussed in greater detail in Appendix C.2.

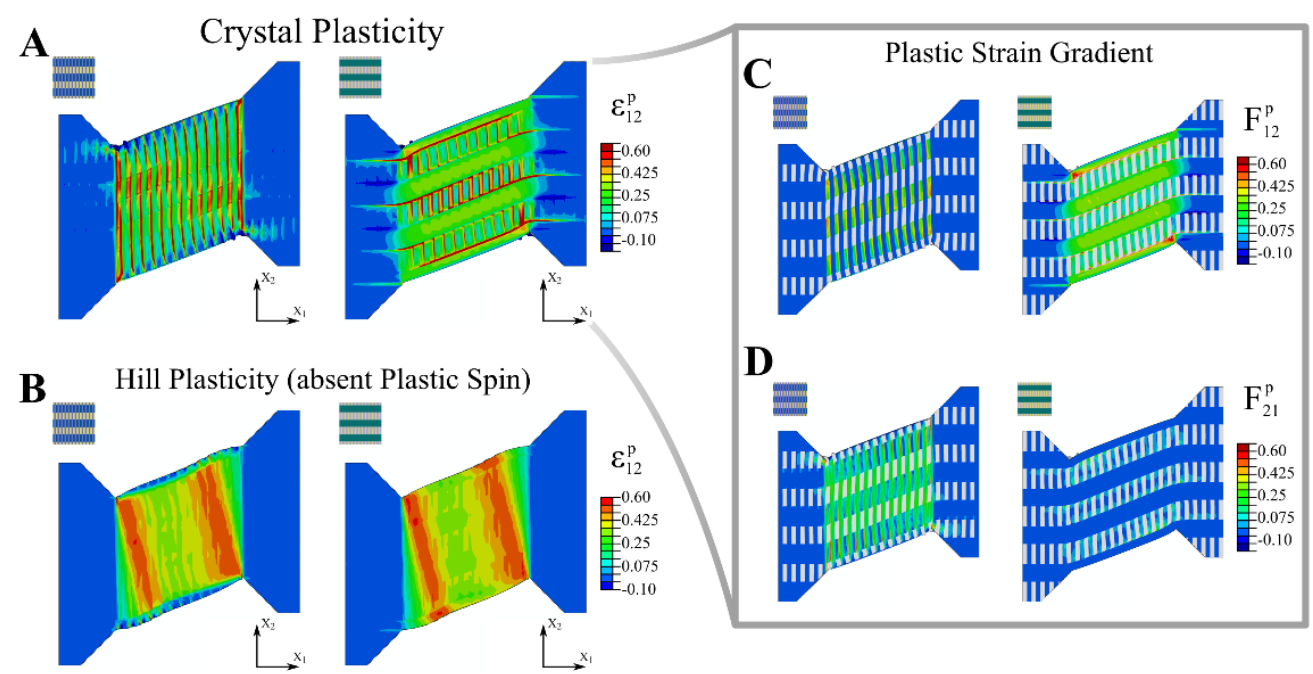

Figure 13: Predictions of the distributions of deformations on the $X_{1}-X_{2}$ mid-plane of the specimen in the $X Z$ orientation at an applied shear strain $\Gamma_{21}=40 \%$. Distributions of the plastic strain $\varepsilon_{12}^{p}$ via the (A) crystal plasticity and (B) Hill plasticity models. Crystal plasticity predictions of the distributions of the deformation gradients (C) $F_{12}^{p}$ and (D) $F_{21}^{p}$ in the tows (matrix pockets shown blank). The specimen sketches use the colour scheme from Fig. 1 and indicate the section ( $X$ or $Z$-section) on which the distributions are shown.

The elastic straining leads to the development of large stresses in the tows as seen in Figs. 14A and 14B, which show spatial distributions of $\sigma_{e}$ in specimens subjected to $\Gamma_{21}=40 \%$ on $Z$ and $X$-sections of the $X Z$ and $Z X$ orientation specimens, respectively. These stresses (resulting from elastic straining) are the source of the predicted hardening response. Moreover, the reverse plasticity is also due to the elastic straining and can be rationalised as follows. Unloading results in elastic recovery of the tows, which in turn reverses plastic deformation within the remainder of the composite (including the matrix pockets) and manifests itself via the observed hysteresis. The underprediction of the level of reverse plasticity in the model compared to measurements could be related to multiple factors, including that the model neglects viscoelastic effects in the matrix and does not account for residual stresses within the composite that are typically generated during manufacturing. 

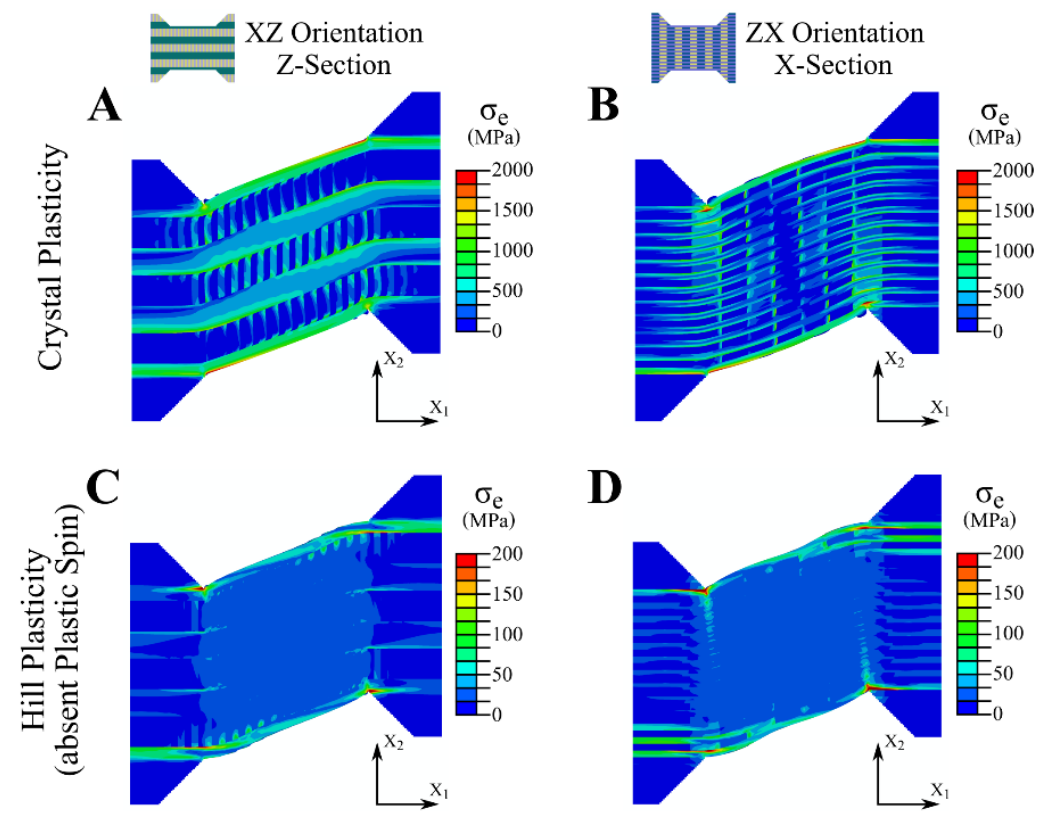

Figure 14: The distributions of the von-Mises effective stress $\sigma_{e}$ on the $X_{1}-X_{2}$ mid-plane of the specimen at an applied shear strain $\Gamma_{21}=40 \%$. The distributions are shown on the $Z$ - and $X$-sections of the specimen for the $X Z$ and $Z X$ orientations, respectively for the crystal plasticity model ((A) and (B) respectively) and the Hill model ((C) and (D) respectively. The specimen sketches at the top use the colour scheme from Fig. 1.

\subsection{Plastic incompatibility and the origin of the hardening response}

We have shown above that the source of the hardening is primarily related to the development of plastic incompatibility between the phases of the 3D composite. This incompatibility, while predicted by the crystal plasticity models for the tows, is not captured at all by the Hill model. This is particularly surprising given that elastic and plastic anisotropy in tow properties are accounted for in a reasonably similar manner in both the Hill and crystal plasticity models, i.e. they have large strengths in the axial tow directions and low tensile and shear strengths in the other directions.

The key to understanding the difference lies in how they incorporate plastic spin $w_{i j}^{p}$. In the Hill model we assume that the plastic spin vanishes with the objective stress rate defined based on the total (material) spin. Conversely, texture evolution is explicitly accounted for in the crystal plasticity model with plastic spin given by Eq. (4.21). Therefore, the elastic spin rate differs from the total (material) spin, which results in differing objective stress rates in the Hill and crystal plasticity models. We shall first discuss the effect of this plastic spin on the crystal plasticity predictions to help clarify the differences between the Hill and crystal plasticity models.

The spatial distributions of $F_{12}^{p}$ and $F_{21}^{p}$ in the tows in the $X$ and $Z$-sections of a $X Z$-orientation specimen at an applied $\Gamma_{21}=40 \%$ are included in Figs. $13 \mathrm{C}$ and $13 \mathrm{D}$, respectively (the matrix pockets are left blank because the matrix is isotropic and not modelled via the crystal plasticity model). The plastic components of the deformation gradient are set by the orientation of the slip systems as given by Eq. (4.19): with these slip system orientations differing in the $X-, Y$ and $Z$-tows, this results in a spatially non-uniform $F_{i j}^{p}$ as well as $F_{i j}^{p} \neq F_{j i}^{p}$ for $i \neq j$ within each phase (tow), i.e. a non-zero plastic spin. Recalling that compatibility requires the total deformation gradient within the gauge section to be approximately spatially uniform, this then implies that $F_{i j}^{*}$ is spatially heterogenous and, correspondingly, so is the spin rate $\dot{w}_{i j}^{*}$. This 
heterogeneity in $\dot{w}_{i j}^{*}$ implies a spatially non-uniform objective stress rate, which in turn leads to an evolution of a spatially heterogenous plastic strain field and the plastic incompatibility discussed above. Thus, the plastic incompatibility is a direct consequence of the fact that in the crystal plasticity model the fibre orientations are rotating with respect to the tow material, i.e. there is a non-zero plastic spin, and that this rotation is different in the differently oriented tows. By contrast, in the Hill model we have neglected plastic spin and thus have assumed that the fibres rotate with the material. This results in the prediction of spatially uniform fields with no plastic incompatibility and no strain hardening. These rotation effects (and the ensuing plastic incompatibility) only become significant at large shear strains, which is why the Hill model accurately captures initial yield but not the large strain behaviour. Thus, given the low failure strains of most conventional 2D composites, the Hill model suffices to describe their plastic behaviour.
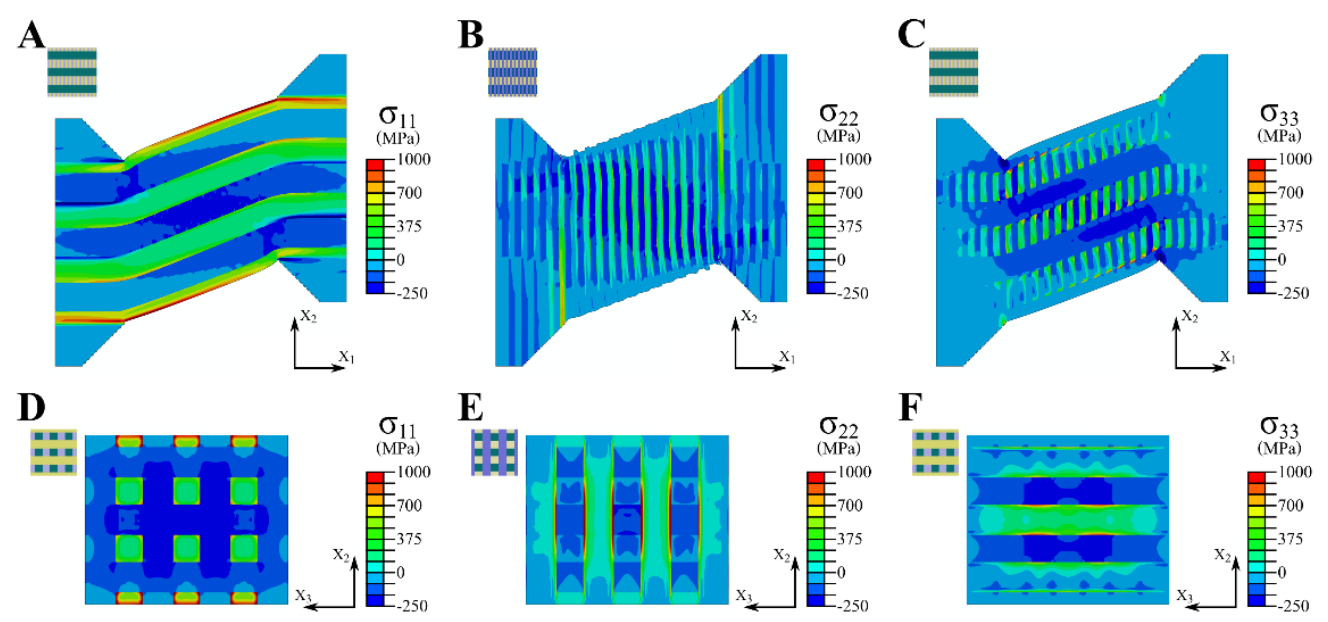

Figure 15: Crystal plasticity predictions of the stress distributions in the $X Z$ orientation specimen at an applied shear strain $\Gamma_{21}=40 \%$. Distribution of $\sigma_{11}$ on the (A) $X_{1}-X_{2}$ mid-plane (Z-section) and (D) $X_{2}-X_{3}$ mid-plane ( $Y$-section), $\sigma_{22}$ on the (B) $X_{1}-X_{2}$ mid-plane ( $X$-section) and (E) $X_{2}-X_{3}$ midplane $\left(X\right.$-section), and $\sigma_{33}$ on the (C) $X_{1}-X_{2}$ mid-plane ( $Z$-section) and (F) $X_{2}-X_{3}$ mid-plane $(Y$ section). Here the $a$-section refers to the tow through which the section was taken, i.e. a $Y$-section means a section through the mid-plane of a $Y$-tow. (A), (B) and (C) show distributions on the deformed configuration while (D), (E) and (F) show distributions on the undeformed configuration. The sketches of the specimen sections use the colour scheme from Fig. 1 and indicate the section on which the distributions are plotted.

The above discussion clarifies that that primary cause of the Hill model not capturing critical features of the large strain shear response of the 3D composites is the assumption of zero plastic spin that is employed here. Additional constitutive rules to specify a non-zero plastic spin can be included in the Hill model (see for example Aravas (1994)), but such rules typically require an understanding of the internal texture of the tows much like what is assumed within the crystal plasticity framework used here.

\subsection{Effect of the $3 D$ tow architecture}

It may be natural to assume that most of the effects discussed above would also be present in traditional 2D laminated composites if delamination failure was somehow prevented (e.g. via Z-pinning; see for example Mouritz (2007)). Here we aim to demonstrate that the 3D tow topology not only plays a role in inhibiting delamination but also strongly influences the hardening response of the composite. 
To quantify the role of $Y$-tows, we include in Fig. 12 predictions of the shear responses of the specimens in the $X Z$ and $Z X$ orientations with the $Y$-tows replaced by the matrix material (all other tow and matrix properties are kept fixed at their reference values with the tow and matrix pressure dependency $\mu=\mu_{m}=0.2$ ). The strain hardening response of the composites is dramatically reduced in the absence of the $Y$-tows even though the shear responses of the matrix pockets and $Y$-tows in the $X_{1}-X_{2}$ planes are expected to be largely similar. To understand this rather counterintuitive effect, let us consider the loading in the $X Z$ orientation specimen with the $Y$-tows present. To better illustrate the stress field, we include in Fig. 16 the distributions of the Cauchy stresses $\sigma_{11}, \sigma_{22}$ and $\sigma_{33}$ on the $X_{1}-X_{2}$ mid-plane (on the $X$ - and $Z$-sections as indicated in the figure) and the $X_{2}-X_{3}$ mid-plane (on the $X$ - and $Y$-sections again as indicated in the figure) of the specimen at an applied shear strain $\Gamma_{21}=40 \%$. These plots show that stresses of approximately equal magnitude develop in all tows. The stresses are primarily tensile in the axial tow direction but the transverse stresses are typically compressive. The large stresses within the $Y$-tows suggest that the tows play two roles:

(i) The tensile axial stresses confirm the role the tows play in preventing the delamination failure that typically occurs during shear loading of a 2D laminated composite.

(ii) The transverse compressive transverse stresses in the tows illustrates their role in generating confining stresses within the composite. This confining stress enables the build-up of pressure in the composite and, combined with the pressure sensitivity of the yield strength, leads to additional hardening.

This $3 \mathrm{D}$ confinement effect is also expected to be present in Z-pinned composites. However, the volume fraction of the Z-pins is usually rather small and this limits their effect (Mouritz, 2007). Increasing the volume fraction of $Z$-pins is usually not done as it induces significant imperfections in the form of fibre waviness. This is because unlike in 3D noobed composites, the $Z$-pins are not integral to the composite lay-up but rather inserted a posteriori.

\section{Concluding remarks}

We have demonstrated that $3 \mathrm{D}$ non-woven carbon fibre composites with an orthogonal tow architecture display a shear response unlike most traditional laminated $2 \mathrm{D}$ composites. In particular, they have a high shear ductility in excess of $50 \%$, a strongly strain hardening response, an unloading modulus that increases with increasing deformation and significant recovery of plastic strain upon unloading. In fact, X-ray tomographic imaging of the specimens suggest there is minimal damage evolution within the specimens even at applied shear strains of $40 \%$. Final fracture of the specimens occurs by tensile fracture of the tows and is accompanied by significant tow pull-out with fracture surfaces similar to those observed in the tensile fracture of unidirectional composites.

Micro-mechanical models were developed wherein all tows were explicitly modelled as anisotropic continua in an attempt to gain a mechanistic understanding of the unusual observations. The Hill anisotropic plasticity model, which is commonly used to model tows in 2D composites, is shown to be unable to capture many critical features of the shear response including the strain hardening and the recovery of plastic strain upon unloading. By contrast, modelling the tows using a crystal plasticity-based framework with pressure dependent yield captured most of the critical observations with excellent fidelity. The underlying reason for this was shown to be related to the fact that the variant of the Hill model considered here neglects texture evolution within the tows, i.e. it assumes zero plastic spin such that the fibres rotate with the material and this assumption leads to significant errors at finite strains. The crystal plasticity-based model was also used to elucidate the effect of the $3 \mathrm{D}$ tow architecture, 
including the role of the orthogonal tows in enhancing the confining stresses and the prevention of delamination failure modes.

The model presented here is intended to be the simplest homogenized model that is capable of capturing the salient physics governing the shear response. Further refinements to include the effects of micro-cracking within the tows, matrix and especially at the interfaces within the different phases as well as accounting for residual stresses induced during manufacture, imperfections such as fibre and tow waviness and the initial cracks along with viscoelastic effects within the matrix are all expected to improve the fidelity of the predictions. Nevertheless, the crystal plasticity-based model has helped give mechanistic insight into the key mechanisms that lead to the novel behaviour of the 3D non-woven composites investigated here. Moreover, given that the all the parameters of the crystal-plasticity model (elastic constants, slip system strength and pressure sensitivity co-efficient) have a direct physical interpretation and can be independently measured, the framework has the capability of serving as a numerical tool to help optimise the topology of such 3D composites. 


\section{Appendix A: Microstructural analysis of the composite}

Specimens were sectioned with a diamond cutting saw and polished to an RMS roughness of $<50 \mathrm{~nm}$ to visualise both the fibre and crack distributions in the tows via optical microscopy. These optical micrographs are shown in Figs. A1A and A1C, which focus on the $X$-tows and $Z$-tows, respectively. The tow level waviness (as seen in the XCT scans in Fig. 3) is reconfirmed in these images, but these higher resolution images also reveal fibre-level waviness within the tows. Fibre directionality analysis was performed with the image processing software ImageJ (developed by the $\mathrm{NIH}$ ), and the resulting histograms from the analysis of ten $X$ and $Z$-tows are included in Fig. A1B and A1D, respectively with the tow direction defined as the fibre angle $\phi=0^{\circ}$. The $X$-tows have a dispersion of fibre angles with a standard deviation $\sigma=2.95^{\circ}$, while the lower fibre volume fraction in the $Z$-tows results in $\sigma=7.84^{\circ}$ in those tows. This suggests that the stiffness knockdown from the ideal stiffness due to fibre waviness is higher for the $Z$-tows. Other imperfections in the as-manufactured 3D composites such as cracks and delaminated areas are seen clearly in the micrographs in Figs. $\mathrm{A} 1 \mathrm{~A}$ and $\mathrm{A} 1 \mathrm{C}$. The cracking exists largely in the matrix pockets and along the interfaces between the phases of the composite.

A

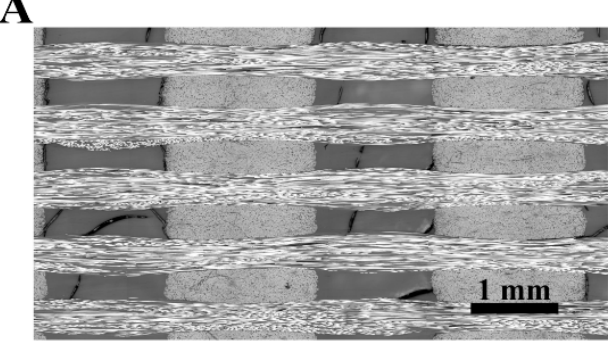

C

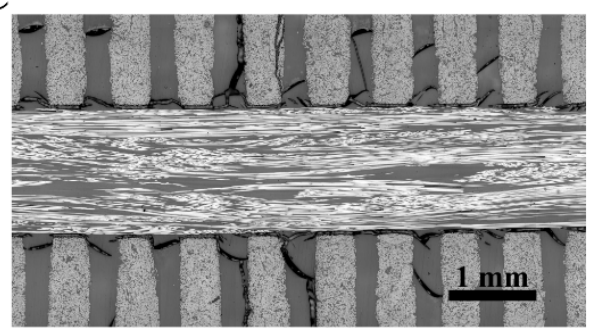

B

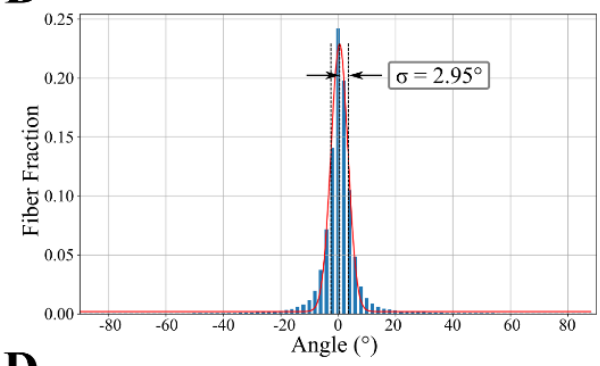

D

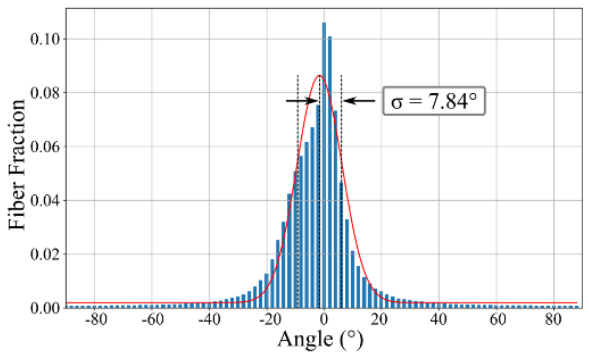

Figure A1: Optical micrograph of sections of the as-manufactured 3D composites focussing on the (A) $X$-tows and (C) $Z$-tows. Corresponding histograms of the dispersion of the fibre orientations are shown for the (B) $X$-tows and (D) $Z$-tows. These histograms were estimated from measurements over ten tows with the tow direction defined as the fibre angle $\phi=0^{\circ}$.

\section{Appendix B: Homogenised elastic constants for the tows}

With $v_{f}$ denoting the fibre volume fraction within the tow (i.e. $v_{f}=V_{f}^{X}$ for the $X$-tow, $v_{f}=$ $V_{f}^{Z}$ for the $Z$-tow etc.) we infer the 5 independent elastic constants as follows. The Young's modulus in the fibre direction is given by a rule of mixtures (Voigt bound) as

$$
E_{\alpha}=E_{f} v_{f}+\left(1-v_{f}\right) E_{m}
$$

while the transverse Young's modulus is inferred from the Halpin-Tsai (Halpin and Kardos, 1976) model as 


$$
E_{\beta}=E_{\gamma}=E_{m} \frac{1+\eta_{E} \xi v_{f}}{1-\eta_{E} v_{f}}
$$

where the parameters $\xi=2+40\left(v_{f}\right)^{10}$ and

$$
\eta_{E}=\frac{E_{f} / E_{m}-1}{E_{f} / E_{m}+\xi} \text {. }
$$

The shear stiffness $G_{\alpha \beta}$ is again calculated from the Halpin-Tsai model via

$$
G_{\alpha \beta}=G_{m} \frac{1+\eta_{G} \xi v_{f}}{1-\eta_{G} v_{f}}
$$

with

$$
\eta_{G}=\frac{G_{f} / G_{m}-1}{G_{f} / G_{m}+\xi}
$$

Here $G_{f} \equiv 0.5 E_{f} /\left(1+v_{f}\right)$ and $G_{m} \equiv 0.5 E_{m} /\left(1+v_{m}\right)$ are the shear moduli of the fibres and the matrix, respectively. The longitudinal Poisson's ratio was determined using a rule of mixtures (Voigt bound) as

$$
v_{\alpha \beta}=v_{f} v_{f}+\left(1-v_{f}\right) v_{m}
$$

while the corresponding Poisson's ratio $v_{\beta \alpha}$ is determined from the symmetry of the elastic compliance as $v_{\beta \alpha}=v_{\alpha \beta}\left(E_{\beta} / E_{\alpha}\right)$. The transverse Poisson's ratio is determined from the model proposed by (Clyne, 1990) as

$$
v_{\beta \gamma}=1-v_{\beta \alpha}-\frac{E_{\beta}}{3 \kappa},
$$

where $\kappa$ is the bulk modulus, which we take to be given by the Reuss bound as

$$
\kappa=\left[\frac{v_{f}}{\kappa_{f}}+\frac{\left(1-v_{f}\right)}{\kappa_{m}}\right]^{-1} .
$$

Here the bulk moduli of the fibres and matrix are given by the usual isotropic relations as $\kappa_{f} \equiv$ $E_{f} /\left[3\left(1-2 v_{f}\right)\right]$ and $\kappa_{m} \equiv E_{m} /\left[3\left(1-2 v_{m}\right)\right]$, respectively.

\section{Appendix C: Hill model for tows}

\section{C.1 Formulation of Hill plasticity absent plastic spin}

In the Hill plasticity model, the total strain rate is written as the sum of the elastic and plastic strain rates such that

$$
\dot{\varepsilon}_{i j}=\dot{\varepsilon}_{i j}^{*}+\dot{\varepsilon}_{i j}^{p}
$$

The elastic strain rate is given by a hypoelastic relation, i.e. the rate form of (4.1) using a Jaumann stress rate with respect to the material spin (which, in keeping with usual implementations of the Hill model, equals the elastic spin as the plastic spin is set identically to zero). The plastic strain rate is assumed to follow an associated flow rule

$$
\dot{\varepsilon}_{i j}^{p}=\dot{\lambda} \frac{\partial \Phi_{H}}{\partial \sigma_{i j}},
$$

in terms of the plastic multiplier $\dot{\lambda}$ and the Hill yield potential $\Phi_{H}$. This potential is specified in terms of the constants $F, G, H, L, M$ and $N$ as

$$
\begin{gathered}
2 \Phi_{H} \equiv F\left(\sigma_{\beta \beta}-\sigma_{\gamma \gamma}\right)^{2}+G\left(\sigma_{\gamma \gamma}-\sigma_{\alpha \alpha}\right)^{2}+H\left(\sigma_{\alpha \alpha}-\sigma_{\beta \beta}\right)^{2} \\
+2 L \sigma_{\beta \gamma}^{2}+2 M \sigma_{\gamma \alpha}^{2}+2 N \sigma_{\alpha \beta}^{2},
\end{gathered}
$$


such that continued plastic flow occurs with $\Phi_{H}=1 / 2$. The six constants $F, G, H, L, M$ and $N$ then follow from six strengths with respect to the principal axes of anisotropy, i.e.

$$
G+H=\frac{1}{\left(Y_{\alpha}\right)^{2}}, \quad F+H=\frac{1}{\left(Y_{\beta}\right)^{2}} \text { and } G+F=\frac{1}{\left(Y_{\gamma}\right)^{2}},
$$

where $Y_{\alpha}, Y_{\beta}$ and $Y_{\gamma}$ are the tensile strengths of the tow in the $\alpha, \beta$ and $\gamma$-directions, respectively (note that the Hill model assumes equal compressive and tensile strengths). Here we take these strengths to be given by the Voigt and Reuss bounds, respectively, such that

$$
Y_{\alpha}=v_{f} \Sigma_{f}+\left(1-v_{f}\right) \Sigma_{m}
$$

and

$$
Y_{\beta}=Y_{\gamma}=\left[\frac{v_{f}}{\Sigma_{f}}+\frac{\left(1-v_{f}\right)}{\Sigma_{m}}\right]^{-1} .
$$

Similarly, the shear strengths $Y_{\beta \gamma}, Y_{\gamma \alpha}$ and $Y_{\alpha \beta}$ give the remaining constants via

$$
L=\frac{1}{2\left(Y_{\beta \gamma}\right)^{2}}, \quad M=\frac{1}{2\left(Y_{\gamma \alpha}\right)^{2}} \text { and } \quad N=\frac{1}{2\left(Y_{\alpha \beta}\right)^{2}},
$$

and in all the calculations reported subsequently we set $Y_{\beta \gamma}=Y_{\gamma \alpha}=Y_{\alpha \beta}=\tau_{m}$, where $\tau_{m}=$ $\Sigma_{m} / \sqrt{3}=10 \mathrm{MPa}$ is the shear strength of the matrix

\section{C.2 Results}

The predictions using the Hill plasticity model for the tows are compared with experimental measurements for the $X Z$ and $Z X$ orientations in Figs. C1A and C1B, respectively. While the Hill model captures the initial yield limit with reasonable accuracy, it predicts a nearly perfectly plastic post-yield response, meaning it does not capture the observed strain hardening behaviour. It also overpredicts the initial shear modulus in both orientations, although this is expected for the same reasons discussed in Section 5.1.

A

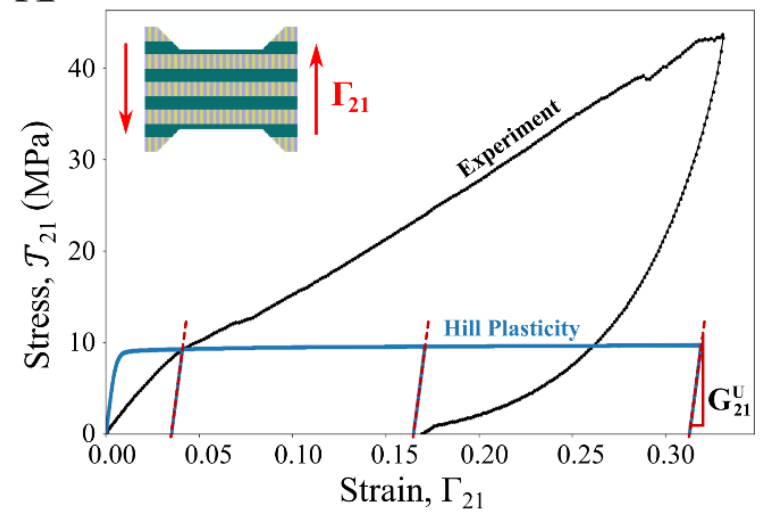

B

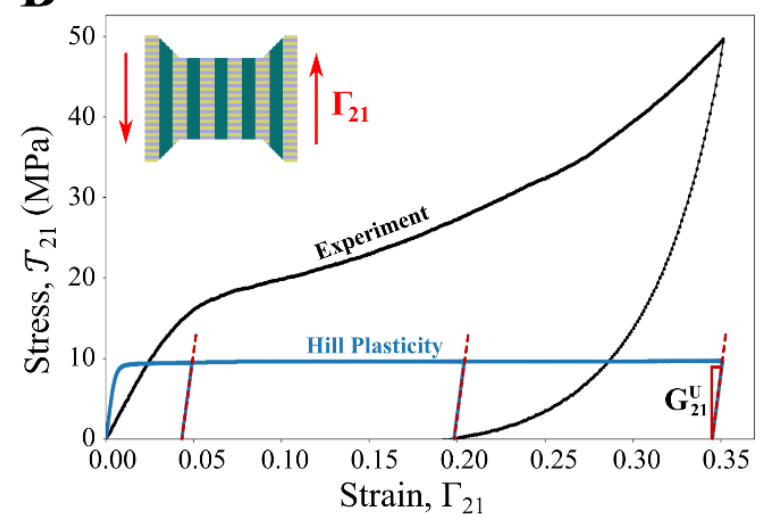

Figure C1: Comparisons between measurements and predictions using the Hill plasticity model for the tows of the shear responses in the (A) $X Z$ and (B) $Z X$ orientations. Predictions include unloading from selected values of the applied shear strains $\Gamma_{21}$. The inset specimen sketches use the colour scheme from Fig. 1.

The inability of the Hill model to capture the hardening, as discussed in Section 5.3, is primarily due to the fact that it does not appropriately account for the evolution of the fibre orientations at large imposed $\Gamma_{21}$. This limitation of the model also manifests in its inability to predict the increase in the unloading stiffness with increasing $\Gamma_{21}$; see Figs. $9 \mathrm{C}$ and $9 \mathrm{D}$ where it is evident 
that the Hill model predicts a constant unloading stiffness over the range of $\Gamma_{21}$ investigated here.

To understand this discrepancy, we include in Fig. 13B predictions of the distribution of plastic strain $\varepsilon_{12}^{p}\left(=\varepsilon_{21}^{p}\right)$ on the $X$ - and $Z$-sections of an $X Z$ orientation specimen at an applied $\Gamma_{21}=$ $40 \%$. In the central gauge section, the plastic strain distributions are nearly uniform despite the considerable heterogeneity and anisotropy in the plastic properties of the individual components of the composite. Given that the total strains within this central gauge section are also spatially uniform (as required by compatibility), no significant elastic strains therefore generated. This absence of elastic straining is illustrated in Fig. 14 where we include predictions of the spatial distributions of von-Mises stress $\sigma_{e}$ in specimens subjected to $\Gamma_{21}=$ $40 \%$. The distributions are shown on the $Z$ - and $X$-sections of the $X Z$ and $Z X$ orientation specimens in Figs. $14 \mathrm{C}$ and $14 \mathrm{D}$, respectively. In the gauge section of the sample there is no significant build-up of axial stresses within the tows, which is consistent with the overall stressstrain response in Fig. C1, implying that continued straining occurs primarily via plastic shear deformation of the tows and the matrix. We thus conclude that the inability of the Hill plasticity model to predict the observed hardening is primarily because it predicts no development of plastic incompatibility between the various phases of the composite.

\section{Acknowledgements}

LRM and VSD are grateful to the Office of Naval Research (ONR) for their financial support through grant number N62909-16-1-2127 on Dynamic performance of 3D assembled composite structures (program managers Dr Joong Kim and Dr Judah Goldwasser). VSD, JMJS and JJCR also acknowledge support from the Netherlands Organisation of Scientific research (NOW) under project 13322 titled "Physics based modelling of failure in textile composites under complex stress states".

\section{References}

Ansar, M., Xinwei, W., Chouwei, Z., 2011. Modeling strategies of 3D woven composites: A review. Compos. Struct. 93, 1947-1963.

Aravas, N., 1994. Finite-strain anisotropic plasticity and the plastic spin. Model. Simul. Mater. Sci. Eng. 2, 483-504.

Arcan, M., Hashin, Z., Voloshin, A., 1978. A method to produce uniform plane-stress states with applications to fiber-reinforced materials. Exp. Mech. 18, 141-146.

Asaro, R.J., 1983. Crystal Plasticity. J. Appl. Mech. 50, 921-934.

Asaro, R.J., Needleman, A., 1985. Texture development and strain hardening in rate dependent polycrystals. Acta Metall. 33, 923-953.

Beissel, S.R., 2014. An Orthotropic Model for Composite Materials in EPIC.

Camanho, P.P., Bessa, M.A., Catalanotti, G., Vogler, M., Rolfes, R., 2013. Modeling the inelastic deformation and fracture of polymer composites-Part II: Smeared crack model. Mech. Mater. $59,36-49$.

Carolan, D., Ivankovic, A., Kinloch, A.J., Sprenger, S., Taylor, A.C., 2017. Toughened carbon fibrereinforced polymer composites with nanoparticle-modified epoxy matrices. J. Mater. Sci. 52, $1767-1788$.

Choi, H., Heinrich, C., Ji, W., 2018. An efficient homogenization technique for fiber tows in textile composites with emphasis on directionally dependent nonlinear stress-strain behavior. Compos. Struct. 208, 816-825.

Clyne, T.W., 1990. A compressibility-based derivation of simple expressions for the transverse Poisson's ratio and shear modulus of an aligned long fibre composite. J. Mater. Sci. Lett. 9, 336-339. 
Cognard, J.Y., Sohier, L., Davies, P., 2011. A modified Arcan test to analyze the behavior of composites and their assemblies under out-of-plane loadings. Compos. Part A Appl. Sci. Manuf. 42, 111-121.

Cox, B.N., Dadkhah, M.S., Inman, R. V., Morris, W.L., Zupon, J., 1992. Mechanisms of compressive failure in 3D composites. Acta Metall. Mater. 40, 3285-3298.

Cox, B.N., Dadkhah, M.S., Morris, W.L., 1996. On the tensile failure of 3D woven composites. Compos. Part A Appl. Sci. Manuf. 27, 447-458.

Cox, B.N., Dadkhah, M.S., Morris, W.L., Flintoff, J.G., 1994. Failure mechanisms of 3D woven composites in tension, compression, and bending. Acta Metall. Mater. 42, 3967-3984.

Dafalias, Y.F., 1984. The plastic spin concept and a simple illustration of its role in finite plastic transformations. Mech. Mater. 3, 223-233.

Daniel, I.M., Luo, J.J., Schubel, P.M., Werner, B.T., 2009. Interfiber/interlaminar failure of composites under multi-axial states of stress. Compos. Sci. Technol. 69, 764-771.

Das, S., Kandan, K., Kazemahvazi, S., Wadley, H.N.G., Deshpande, V.S., 2018. Compressive response of a 3D non-woven carbon-fibre composite. Int. J. Solids Struct. 136, 137-149.

de Sousa Junior, R.R., Gouveia, J.R., Ito, N.M., dos Santos, D.J., 2017. Failure prediction of hybrid composite using Arcan's device and Drucker-Prager model. Polym. Test. 58, 256-261.

Drucker, D.C., Prager, W., 1952. Soil mechanics and plastic analysis or limit design. Q. Appl. Math. $10,157-165$.

Evans, A.G., Adler, W.F., 1978. Kinking as a mode of structural degradation in carbon fiber composites. Acta Metall. 26, 725-738.

Farley, G.L., Jones, R.M., 1992. Crushing Characteristics of Continuous Fiber-Reinforced Composite Tubes. J. Compos. Mater. 26, 37-50.

González, C., LLorca, J., 2007. Mechanical behavior of unidirectional fiber-reinforced polymers under transverse compression: Microscopic mechanisms and modeling. Compos. Sci. Technol. 67, 2795-2806.

Halpin, J.C., Kardos, J.L., 1976. The Halpin-Tsai Equations: A Review. Polym. Eng. Sci. 16, 344 352.

Hasanyan, A.D., Waas, A.M., 2018. Localization in anisotropic elastoplastic micropolar media: Application to fiber reinforced composites. J. Mech. Phys. Solids 121, 1-22.

Hashin, Z., 1980. Failure Criteria for Unidirectional Fibre Composites. J. Appl. Mech. 47, 329-334.

Hill, R., 1948. A theory of the yielding and plastic flow of anisotropic metals. Proc. R. Soc. London 193, 281-297.

Hill, R., Rice, J.R., 1972. Constitutive analysis of elastic-plastic crystals at arbitrary strain. J. Mech. Phys. Solids 20, 401-413.

Hine, P.J., Duckett, R.A., Kaddour, A.S., Hinton, M.J., Wells, G.M., 2005. The effect of hydrostatic pressure on the mechanical properties of glass fibre/epoxy unidirectional composites. Compos. Part A Appl. Sci. Manuf. 36, 279-289.

Huang, H., Waas, A.M., 2009. Compressive response of Z-pinned woven glass fiber textile composite laminates: Modeling and computations. Compos. Sci. Technol. 69, 2338-2344.

Huang, T., Wang, Y., Wang, G., 2017. Review of the Mechanical Properties of a 3D Woven Composite and Its Applications. Polym. Plast. Technol. Eng. 0, 1-17.

Hull, D., Clyne, T.W., 1996. An Introduction to Composite Materials, 2nd Editio. ed. Cambridge University Press.

Jacob, G.C., Fellers, J.F., Simunovic, S., Starbuck, J.M., 2002. Energy absorption in polymer composites for automotive crashworthiness. J. Compos. Mater. 36, 813-850.

Jelf, P.M., Fleck, N.A., 1992. Compression Failure Mechanisms in Unidirectional Composites. J. Compos. Mater. 26, 2706-2726.

Kamiya, R., Cheeseman, B.A., Popper, P., Chou, T.W., 2000. Some recent advances in the fabrication and design of three-dimensional textile preforms: A review. Compos. Sci. Technol. 60, 33-47.

Kazemahvazi, S., Khokar, N., Hallstrom, S., Wadley, H.N.G., Deshpande, V.S., 2016. Confluent 3Dassembly of fibrous structures. Compos. Sci. Technol. 127, 95-105.

Khokar, N., 2012. 3D fabric and a method and apparatus for producing such a 3D fabric. 9797076B2.

Khokar, N., 2002. Noobing: A Nonwoven 3D Fabric-forming Process Explained. J. Text. Inst. 93, $52-74$. 
Khokar, N., 2001. 3D-Weaving: Theory and Practice. J. Text. Inst. 92, 193-207.

Khokar, N., 1997. 3D-weaving and noobing: characterization of interlaced and non-interlaced 3D fabric forming principles. Chalmers University of Technology.

Lafarie-Frenot, M.C., Touchard, F., 1994. Comparative in-plane shear behaviour of long-carbon-fibre composites with thermoset or thermoplastic matrix. Compos. Sci. Technol. 52, 417-425.

Liu, B.G., Kandan, K., Wadley, H.N.G., Deshpande, V.S., 2018. Deep penetration of ultra-high molecular weight polyethylene composites by a sharp-tipped punch. J. Mech. Phys. Solids In Press.

Llorca, J., González, C., Molina-Aldareguía, J.M., Segurado, J., Seltzer, R., Sket, F., Rodríguez, M., Sádaba, S., Muñoz, R., Canal, L.P., 2011. Multiscale modeling of composite materials: A roadmap towards virtual testing. Adv. Mater. 23, 5130-5147.

Mandel, U., Taubert, R., Hinterhölzl, R., 2015. Mechanism based nonlinear constitutive model for composite laminates subjected to large deformations. Compos. Struct. 132, 98-108.

McGlockton, M.A., Cox, B.N., McMeeking, R.M., 2003. A Binary Model of textile composites: III high failure strain and work of fracture in 3D weaves. J. Mech. Phys. Solids 51, 1573-1600.

Mouritz, A.P., 2007. Review of z-pinned composite laminates. Compos. Part A Appl. Sci. Manuf. 38, $2383-2397$.

Mouritz, A.P., Bannister, M.K., Falzon, P.J., Leong, K.H., 1999. Review of applications for advanced three-dimensional fibre textile composites. Compos. Part A Appl. Sci. Manuf. 30, 1445-1461.

Mouritz, A.P., Gellert, E., Burchill, P., Challis, K., 2001. Review of advanced composite structures for naval ships and submarines. Compos. Struct. 53, 21-24.

Naya, F., Gonzalez, C., Lopes, C.S., Van der Veen, S., Pons, F., 2017. Computational micromechanics of the transverse and shear behavior of unidirectional fiber reinforced polymers including environmental effects. Compos. Part A Appl. Sci. Manuf. 92, 146-157.

Naya, F., Herraez, M., Gonzalez, C., Lopes, C.S., Veen, S. Van der, Pons, F., 2017. Computational micromechanics of fiber kinking in unidirectional FRP under different environmental conditions. Compos. Sci. Technol. 144, 26-35.

Paepegem, W. Van, Baere, I. De, Degrieck, J., 2006. Modelling the nonlinear shear stress and strain response of glass fibre-reinforced composites. Part I: Experimental results. Compos. Sci. Technol. 66, 1455-1464.

Petriccione, A., Annicchiarico, D., Antonucci, V., Giordano, M., Riccio, A., Scaramuzzino, F., Zarrelli, M., 2012. A stiffness volume averaging based approach to model non-crimp fabric reinforced composites. Compos. Sci. Technol. 72, 360-369.

Pinho, S.T., Dávila, C.G., Camanho, P.P., Iannucci, L., Robinson, P., 2005. Failure Models and Criteria for FRP Under In-Plane or Three-Dimensional Stress States Including Shear Nonlinearity. NASA Tech. Rep.

Poe, C.C., Dexter, H.B., Raju, I.S., 1999. Review of the NASA Textile Composites Research. J. Aircr. 36, 876-884.

Puck, A., Schürmann, H., 1998. Failure analysis of FRP laminates by means of physically based phenomenological models. Compos. Sci. Technol. 58, 1045-1067.

R. Wisnom, M., 1995. The effect of fibre rotation in +-45 degree tension tests on measured shear properties. Composites 26, 25-32.

Rabinowitz, S., Ward, I.M., Parry, J.S.C., 1970. The effect of hydrostatic pressure on the shear yield behaviour of polymers. J. Mater. Sci. 5, 29-39.

Sauer, J.A., 1977. Deformation, yield and fracture of polymers at high pressure. Polym. Eng. Sci. 17, $150-164$.

Schmidt, S., Beyer, S., Knabe, H., Immich, H., Meistring, R., Gessler, A., 2004. Advanced ceramic matrix composite materials for current and future propulsion technology applications. Acta Astronaut. 55, 409-420.

Shin, E.S., Pae, K.D., 1992. Effects of Hydrostatic Pressure on In-Plane Shear Properties of Graphite/Epoxy Composites. J. Compos. Mater. 26, 828-868.

Song, S., Waas, A.M., Shahwan, K.W., Xiao, X., Faruque, O., 2007. Braided textile composites under compressive loads: Modeling the response, strength and degradation. Compos. Sci. Technol. 67, 3059-3070.

Sun, C.T., Zhu, C., 2000. The effect of deformation-induced change of fiber orientation on the non- 
linear behavior of polymeric composite laminates. Compos. Sci. Technol. 60, 2337-2345.

Tan, P., Tong, L., Steven, G.P., Ishikawa, T., 2000. Behavior of 3D orthogonal woven CFRP composites. Part I. Experimental investigation. Compos. Part A Appl. Sci. Manuf. 31, 259-271.

Tan, W., Falzon, B.G., 2016. Modelling the nonlinear behaviour and fracture process of AS4/PEKK thermoplastic composite under shear loading. Compos. Sci. Technol. 126, 60-77.

Tao, J.X., Sun, T.C., 1996. Effect of matrix cracking on stiffness of composite laminates. Mech. Compos. Mater. Struct. 3, 225-239.

Tong, L., Mouritz, A.P., Bannister, M.K., 2002. 3D Fibre Reinforced Polymer Composites, Elsevier.

Umer, R., Alhussein, H., Zhou, J., Cantwell, W.J., 2017. The mechanical properties of 3D woven composites. J. Compos. 51, 1703-1716.

Vogler, M., Rolfes, R., Camanho, P.P., 2013. Modeling the inelastic deformation and fracture of polymer composites-Part I: Plasticity model. Mech. Mater. 59, 50-64.

Vogler, T.J., Kyriakides, S., 1999. On the axial propagation of kink bands in fiber composites : Part I experiments. Int. J. Solids Struct. 36, 557-574.

Vyas, G.M., Pinho, S.T., Robinson, P., 2011. Constitutive modelling of fibre-reinforced composites with unidirectional plies using a plasticity-based approach. Compos. Sci. Technol. 71, 10681074.

Ward, I.M., 1971. Review: The yield behaviour of polymers. J. Mater. Sci. 6, 1397-1417.

Yudhanto, A., Lubineau, G., Ventura, I.A., Watanabe, N., Iwahori, Y., Hoshi, H., 2015. Damage characteristics in 3D stitched composites with various stitch parameters under in-plane tension. Compos. Part A Appl. Sci. Manuf. 71, 17-31.

Zhang, P., Gao, Z., Zhang, Q., Khattab, A., Li, G., 2018. Fracture behavior characterization of arcan polycaprolactone based polymer composites prepared by polymerization induced phases separation. Polym. Compos. 